\title{
A Hybrid Intuitionistic Logic: Semantics and Decidability
}

\author{
Rohit Chadha $^{1} \quad$ Damiano Macedonio ${ }^{2}$ Vladimiro Sassone ${ }^{2}$ \\ ${ }^{1}$ Center for Logic and Computation, IST Lisboa \\ ${ }^{2}$ University of Sussex
}

\begin{abstract}
An intuitionistic, hybrid modal logic suitable for reasoning about distribution of resources was introduced in $[17,18]$. The modalities of the logic allow validation of properties in a particular place, in some place and in all places. We give a sound and complete Kripke semantics for the logic extended with disjunctive connectives. The extended logic can be seen as an instance of Hybrid IS5. We also give a sound and complete birelational semantics, and show that it enjoys the finite model property: if a judgement is not valid in the logic, then there is a finite birelational counter-model. Hence, we prove that the logic is decidable.
\end{abstract}

Keywords: spatial distribution of resources, spatial modalities, Kripke and birelational semantics, soundness and completeness, finite-model property.

\section{Introduction}

In current computing, paradigm distributed resources spread over and shared amongst different nodes of a computer system are very common. For example, printers may be shared in local area networks, or distributed data may store documents in parts at different locations. The traditional reasoning methodologies are not easily scalable to these systems as they may lack implicitly trust-able objects such as a central control.

This has resulted in the innovation of several reasoning techniques. A popular approach in the literature has been the use of algebraic systems such as process algebra $[10,21,16]$. These algebras have rich theories in terms of semantics [21], logics [9, 8, 15, 23], and types [16]. Another approach is logic-oriented [17, 18, 38, 22, 39, 31]: intuitionistic modal logics are used as foundations of type systems by exploiting the propositions-as-types, proofs-as-programs paradigm [13]. An instance of this was introduced in [17, 18], and the logic introduced there is the focus of our study.

The formulae in the logic use the standard intuitionistic conjunctive connectives $\wedge$ and $T$, and the intuitionistic implication $\rightarrow$. They also include names, called places. Assertions in the logic are associated with places, and are validated in places. In addition to considering whether a formula is true, we are also interested in where a formula is true. In order to achieve this, the logic has three modalities. The modalities allow us to infer whether a property is validated in a specific place of

Research partially supported by 'DisCo: Semantic Foundations of Distributed Computation', EU IHP 'Marie Curie' project HPMT-CT-2001-00290, by 'MIKADO: Mobile Calculi based on Domains', EU FET-GC project IST-2001-32222, and by 'MyThS: Models and Types for Security in Mobile Distributed Systems', EU FET-GC project IST-2001-32617. The research was carried out when Rohit Chadha was a research fellow at University of Sussex. 
the system (@p), or in an unspecified place of the system $(\diamond)$, or in any part of the system $(\square)$. The modality @ $p$ internalises the model in the logic, and hence the logic can be classified as a hybrid logic $[1,2,4,5,6,7,28,29]$.

A natural deduction for the logic is given in $[17,18]$, and the judgements in the logic mention the places under consideration. The rules for $\diamond$ and $\square$ resemble those for existential and universal quantification of first-order intuitionistic logic. We extend the logic with disjunctive connectives, and extend the natural deduction system to account for these. The deduction system is essentially a conservative extension of propositional intuitionistic logic; and it is in this sense that we will use the adjective "intuitionistic" for the extended logic throughout the paper.

As noted in $[17,18]$, the logic can also be used to reason about distribution of resources in addition to serving as the foundation of a type system. The papers [17, 18], however, lack a model to match the usage of the logic as a tool to reason about distributed resources. In this paper, we bridge the gap by presenting a Kripke-style semantics [20] for the logic extended with disjunctive connectives. In Kripke-style semantics, formulae are considered valid if they remain valid when the atoms mentioned in the formulae change their value from false to true. This is achieved by using a partially ordered set of possible states. Informally, more atoms are true in larger states.

We extend the Kripke semantics of the intuitionistic logic [20], enriching each possible state with a set of places. The set of places in Kripke states are not fixed, and different possible Kripke states may have different set of places. However, the set of places vary in a conservative way: larger Kripke states contain larger set of places. In each possible state, different places satisfy different formulae. In the model, we interpret atomic formulae as resources of a distributed system, and placement of atoms in a possible state corresponds to the distribution of resources.

The enrichment of the model with places reveals the true meaning of the modalities in the logic. The modality @ $p$ expresses a property in a named place. The modality $\square$ corresponds to a weak form of spatial universal quantification and expresses a property common to all places, and the modality $\checkmark$ corresponds to a weak form of spatial existential quantification and expresses a property valid somewhere in the system. For the intuitionistic connectives, the satisfaction of formulae at a place in a possible state follows the standard definition [20].

To give semantics to a logical judgement, we allow models with more places than those mentioned in the judgement. This admits the possibility that a user may be aware of only a certain subset of names in a distributed system. This is crucial in the proof of soundness and completeness as it allows us to create witnesses for the existential $(\diamond)$ and the universal $(\square)$ modalities. The Kripke semantics reveals that the extended logic can be seen as the hybridisation of the well-known intuitionistic modal system IS5 [12, 24, 27, 30, 35, 36].

Following [12, 27, 35, 36], we also introduce a sound and complete birelational semantics for the logic. The reason for introducing birelational semantics is that it allows us to prove decidability. Birelational semantics typically enjoy the finite model property [25, 36]: if a judgement is not provable, then there is a finite counter-model. On the other hand Kripke semantics do not satisfy the finite model property [25, 36]. As in Kripke models, birelational models have a partially ordered set. The elements of this set are called worlds. In addition to the partial order, birelational models also have an equivalence relation amongst worlds, called the accessibility or reachability relation. Unlike the Kripke semantics, we do not enrich each world with a set of places. Instead, we have a partial function, the evaluation function, which attaches a name to a world in its domain. As we shall see, the partiality of the function is crucial to the proof of decidability.

The partial evaluation function must satisfy two important properties. One, coherence, states that if the function associates a name to a world then it also associates the same name to all larger states. The other, uniqueness, states that two different worlds accessible from one another do not evaluate to 
the same name. Coherence is essential for ensuring monotonicity of the logical connective @ $p$, and uniqueness is essential for ensuring the soundness of introduction of conjunction and implication.

Following [36], we also introduce an encoding of the Kripke models into birelational models. The encoding maps a place in a Kripke state into a world of the corresponding birelational model. The encoding ensures that if a formula is validated at a place in a state of the Kripke model, then it is also validated at the corresponding world. The encoding allows us to conclude soundness of Kripke semantics from soundness of birelational semantics. It also allows us to conclude completeness of the birelational models from completeness of Kripke semantics. We emphasise here that any birelational model resulting from the encoding is restricted in the sense that any two worlds reachable from each other are not related in the partial order. Therefore, the finite model property may fail for Kripke semantics even if it holds for birelational models. Birelational semantics gives us more models, and the fact that worlds reachable from each other can also be ordered is essential to achieve finite model property for birelational semantics (see $\S 3.2, \S 5.3$ and $[25,36]$ ).

Surprisingly, the soundness of the birelational models was not straightforward. The problematic cases are the inference rules for introduction of $\square$ and the elimination of $\diamond$. In Kripke semantics, soundness is usually proved by duplicating places in a conservative way [7, 36]. The partiality of the evaluation function, along with the coherence and uniqueness conditions however impeded in obtaining such a result. It has been noted in [36] that the soundness is also non-trivial in the case of birelational models for intuitionistic modal logic. However, the problems with soundness here arise purely because of the hybrid nature of the logic. Soundness is obtained by using a mathematical construction that creates a new birelational model from a given one. In the new model, the set of worlds consists of the reachability relation of the old model, and we add new worlds to witness the existential and universal properties.

The proof of completeness follows standard techniques from intuitionistic logics, and given a judgement that is not provable in the logic we construct a canonical Kripke model that invalidates the judgement. However, following [36], the construction of this model is done in a careful way so that it assists in the proof of decidability. The encoding of Kripke models into birelational models gives us a canonical birelational model. The worlds of canonical birelational models consists of triples: a finite set of places $Q$, a finite set of sentences $\Delta$, and a special place $q$ which is the evaluation of the world.

The set of worlds in the canonical birelational models may be infinite. We show that by identifying the worlds in the birelational model up-to renaming of places, we can construct an equivalent finite model, called the quotient model. This allows us to deduce the finite model property for the birelational semantics, and hence decidability of the logic. The proof is adapted from the case of intuitionistic modal logic [36]. The partiality of the evaluation function is crucial in the proof.

The rest of the paper is organised as follows. In $\S 2$, we introduce the logic and the Kripke semantics. In $\S 3$, we introduce the birelational semantics, and prove the soundness of the logic with respect to birelational models. The encoding of Kripke models into birelational models is also given and it allows us to conclude soundness of Kripke semantics. The construction of canonical models and completeness is discussed in $\S 4$. In $\S 5$, we construct the quotient model and prove the finite model property for birelational models. Related work is discussed in $\S 6$, and our results are summarised in $\S 7$. For the sake of a smooth exposition, we have omitted some standard proofs, which are detailed in a companion technical report [11]. 


\section{Logic}

We now introduce, through examples, the logic presented in $[17,18]$ extended with disjunctive connectives, thus giving us the full set of intuitionistic connectives. The logic can be used to reason about heterogeneous distributed systems. To gain some intuition, consider a distributed peer to peer database where the information is partitioned over multiple communicating nodes (peers).

Informally, the database has a set of nodes, or places, and a set of resources (data) distributed amongst these places. The nodes are chosen from the elements of a fixed set, denoted by $p, q, r, s, \ldots$ Resources are represented by atomic formulae $A, B, \ldots \in$ Atoms. Intuitively, an atom $A$ is valid in a place $p$ if that place can access the resource identified by $A$.

Were we reasoning about a particular place, the logical connectives of the intuitionistic framework would be sufficient. For example, assume that a particular document, doc, is partitioned in two parts, $d_{0} c_{1}$ and $d_{0} c_{2}$, and in order to gain access to the document a place has to access both of its parts. This can be formally expressed as the logical formula: $\left(\operatorname{doc}_{1} \wedge \operatorname{doc}_{2}\right) \rightarrow$ doc, where $\wedge$ and $\rightarrow$ are the logical conjunction and implication. If $\mathrm{doc}_{1}$ and $\mathrm{doc}_{2}$ are stored in a particular place, then the usual intuitionistic rules allow one to infer that the place can access the entire document.

The intuitionistic framework is extended in [18] to reason about different places. An assertion in such a logic takes the form " $\varphi$ at $p$ ", meaning that formula $\varphi$ is valid at place $p$. The construct "at" is a meta-linguistic symbol and points to the place where the reasoning is located. For example, $\operatorname{doc}_{1}$ at $p$ and $\operatorname{doc}_{2}$ at $p$ formalise the notion that the parts $\operatorname{doc}_{1}$ and $\operatorname{doc}_{2}$ are located at the node $p$. If, in addition, the assertion $\left(\left(\operatorname{doc}_{1} \wedge \mathrm{doc}_{2}\right) \rightarrow\right.$ doc $) \boldsymbol{a t} p$ is valid, we can conclude that the document doc is available at $p$.

The logic is a conservative extension of intuitionistic logic in the sense that if we restrict our attention to formulae without modalities then the 'local' proof system in a single place $p$ mimics the standard intuitionistic one. For instance, the deduction described above is formally

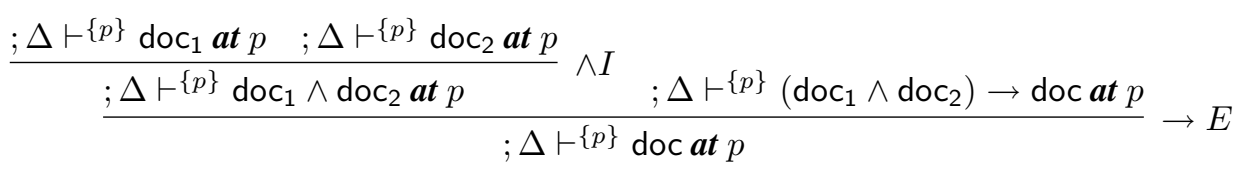

where $\Delta \stackrel{\text { def }}{=}\left(\operatorname{doc}_{1} \wedge \operatorname{doc}_{2}\right) \rightarrow \operatorname{doc}$ at $p, \operatorname{doc}_{1}$ at $p, \operatorname{doc}_{2}$ at $p$. It is easy to see that this derivation becomes a standard intuitionistic one if rewritten without the 'place construct' at $p$.

In the assertion $\varphi$ at $p, \varphi$ will not contain any occurrences of the construct at. Instead, $\varphi$ will use modalities @ $p$, one for each place in the system, to cast the meta-linguistic at at the language level. A modality @ internalises resources at the location $p$, and the modal formula $\varphi @ p$ means that the property $\varphi$ is valid at $p$, and not necessarily anywhere else. Indeed both $\varphi$ at $p$ and $\varphi @ p$ will have the same semantics, and it is possible to define an equivalent logic in which the construct at is not needed. However, we will prefer to keep the distinction in the logic as this was the case in $[17,18]$. The introduction and elimination rules for the modality @ are also more elegant if we maintain this distinction. We need to keep track of where the reasoning is happening, and therefore if we confuse at with @ then we will always need sentences of the form $\varphi @ p$. In that case @-elimination could be applied only when the formula has two or more occurrences of @, namely only when it is of the form $\varphi @ p @ q$.

An assertion of the form $\varphi @ p a t p^{\prime}$ means that we are located at the place $p^{\prime}$, and we are reasoning about the property $\varphi$ that is validated at place $p$. For example, suppose that the place $p$ has the first half of the document, i.e., $\operatorname{doc}_{1} a t p$, and $p^{\prime}$ has the second one, i.e., $\operatorname{doc}_{2} \boldsymbol{a t} p^{\prime}$. In the logic we can formalise the fact that $p^{\prime}$ can send the part doc 2 to $p$ by using the assertion $\left(\operatorname{doc}_{2} \rightarrow\left(\operatorname{doc}_{2} @ p\right)\right)$ at $p^{\prime}$. 
The rules of the logic will conclude $\mathrm{doc}_{2}$ at $p$ and so doc at $p$. The formal derivation, (if we look ahead at the rules in Fig. 1), is

$$
\frac{; \Delta \vdash\left\{p, p^{\prime}\right\} \mathrm{doc}_{2} \text { at } p^{\prime} ; \Delta \vdash^{\left\{p, p^{\prime}\right\}}\left(\operatorname{doc}_{2} \rightarrow\left(\operatorname{doc}_{2} @ p\right)\right) \text { at } p^{\prime}}{\frac{; \Delta \vdash^{\left\{p, p^{\prime}\right\}}\left(\operatorname{doc}_{2} @ p\right) \text { at } p^{\prime}}{; \Delta \vdash^{\left\{p, p^{\prime}\right\}} \mathrm{doc}_{2} \text { at } p} @ E} \rightarrow E
$$

Where $\Delta \stackrel{\text { def }}{=} \operatorname{doc}_{2}$ at $p,\left(\operatorname{doc}_{2} \rightarrow\left(\operatorname{doc}_{2} @ p\right)\right)$. The assertion doc at $p$ can then be derived by enriching $\Delta$ with the assumptions $\operatorname{doc}_{1}$ at $p,\left(\operatorname{doc}_{1} \wedge \operatorname{doc}_{2}\right) \rightarrow$ doc at $p$, and by mimicking the derivation (1).

The logic also has two other modalities to accommodate reasoning about properties valid at different locations, which we discuss briefly. Knowing exactly where a property holds is a strong ability, and we may only know that the property holds somewhere without knowing the specific location where it holds. To deal with this, the logic has the modality $\diamond$ : the formula $\diamond \varphi$ means that $\varphi$ holds in some place of the system. In the example above, the location of $d_{0} c_{2}$ is not important as long as we know that this document is located in some place from where it can be sent to $p$. Formally, this can be expressed by the logical formula $\diamond\left(\operatorname{doc}_{2} \wedge\left(\operatorname{doc}_{2} \rightarrow\left(\operatorname{doc}_{2} @ p\right)\right)\right)$ at $p^{\prime}$. By assuming this formula, we can infer $\mathrm{doc}_{2}$ at $p$, and hence the document doc is available at $p$. We will illustrate this inference in the deduction system at the end of the section (see Example 1).

Even if we deal with resources distributed in heterogeneous places, certain properties are valid everywhere. For this purpose, the logic has the modality $\square$ : the formula $\square \varphi$ means that $\varphi$ is valid everywhere. In the example above, $p$ can access the document doc if there is a place that has the part $\mathrm{doc}_{2}$ and can send it everywhere. This can be expressed by the formula $\diamond\left(\operatorname{doc}_{2} \wedge\left(\operatorname{doc}_{2} \rightarrow\right.\right.$ $\left.\square \mathrm{doc}_{2}\right)$ ) at $p^{\prime}$. The rules of the logic would allow us to conclude that $\mathrm{doc}_{2}$ is available at $p$. Therefore the document doc is also available at $p$. We will illustrate this inference at the end of the section (see Example 2).

We now define the logic formally. As mentioned above, it is essentially the logic introduced in [18] enriched with the disjunctive connectives $\vee$ and $\perp$. This allows us to express properties such as: the document $\mathrm{doc}_{2}$ is located either at $p$ itself or at $q$ (in which case $p$ has to fetch it). This can be expressed by the formula $\left(\operatorname{doc}_{2} \vee\left(\left(\operatorname{doc}_{2} @ q\right) \rightarrow \operatorname{doc}_{2}\right)\right)$ at $p$.

For the rest of the paper, we shall assume a fixed countable set of atomic formulae Atoms, and we vary the set of places. Given a countable set of places $P l$, let $F r m(P l)$ be the set of formulae built from the following grammar:

$$
\varphi::=A|\top| \perp|\varphi \wedge \varphi| \varphi \vee \varphi|\varphi \rightarrow \varphi| \varphi @ p|\square \varphi| \nabla \varphi .
$$

Here the syntactic category $p$ stands for elements from $P l$, and the syntactic category $A$ stands for elements from Atoms. The elements in $\operatorname{Frm}(\mathrm{Pl})$ are said to be pure formulae, and are denoted by small Greek letters $\varphi, \psi, \mu \ldots$ An assertion of the form $\varphi$ at $p$ is called sentence. We denote by capital Greek letters $\Gamma, \Gamma_{1}, \ldots$ (possibly empty) finite sets of pure formulae, and by capital Greek letters $\Delta, \Delta_{1}, \ldots$ (possibly empty) finite sets of sentences.

Each judgement in this logic is of the form

$$
\Gamma ; \Delta \vdash^{P} \varphi \text { at } p
$$

where

- The global context $\Gamma$ is a (possibly empty) finite set of pure formulae, and represents the properties assumed to hold at every place of the system. 


$$
\begin{aligned}
& \overline{\Gamma ; \Delta, \varphi \boldsymbol{a t} p \vdash^{P} \varphi \boldsymbol{a t} p} L \\
& \overline{\Gamma, \varphi ; \Delta \vdash^{P} \varphi \text { at } p} G \\
& \overline{\Gamma ; \Delta \vdash^{P} \top \boldsymbol{a t} p}{ }^{\top I} \\
& \frac{\Gamma ; \Delta \vdash^{P} \perp \text { at } p}{\Gamma ; \Delta \vdash^{P} \psi \text { at } p} \perp E \\
& \frac{\Gamma ; \Delta \vdash^{P} \varphi_{1} \text { at } p}{\Gamma ; \Delta \vdash^{P} \varphi_{1} \vee \varphi_{2} \text { at } p} \vee I_{1} \\
& \frac{\Gamma ; \Delta \vdash^{P} \varphi_{2} \text { at } p}{\Gamma ; \Delta \vdash^{P} \varphi_{1} \vee \varphi_{2} \text { at } p} \vee I_{2} \\
& \frac{\Gamma ; \Delta \vdash^{P} \varphi_{1} \vee \varphi_{2} \text { at } p \quad \Gamma ; \Delta, \varphi_{1} \text { at } p \vdash^{P} \psi \text { at } p \quad \Gamma ; \Delta, \varphi_{2} \text { at } p \vdash^{P} \psi \boldsymbol{a t} p}{\Gamma ; \Delta \vdash^{P} \psi \text { at } p} \vee E \\
& \frac{\Gamma ; \Delta \vdash^{P} \varphi_{i} \text { at } p \quad i=1,2}{\Gamma ; \Delta \vdash^{P} \varphi_{1} \wedge \varphi_{2} \text { at } p} \wedge I \quad \frac{\Gamma ; \Delta \vdash^{P} \varphi_{1} \wedge \varphi_{2} \text { at } p}{\Gamma ; \Delta \vdash^{P} \varphi_{i} \text { at } p} \wedge E_{i} \quad(i=1,2) \\
& \frac{\Gamma ; \Delta, \varphi \text { at } p \vdash^{P} \psi \text { at } p}{\Gamma ; \Delta \vdash^{P} \varphi \rightarrow \psi \text { at } p} \rightarrow I \quad \frac{\Gamma ; \Delta \vdash^{P} \varphi \rightarrow \psi \text { at } p \quad \Gamma ; \Delta \vdash^{P} \varphi \text { at } p}{\Gamma ; \Delta \vdash \psi \text { at } p} \rightarrow E \\
& \frac{\Gamma ; \Delta \vdash^{P} \varphi \text { at } p}{\Gamma ; \Delta \vdash^{P} \varphi @ p \text { at } p^{\prime}} @ I \quad \frac{\Gamma ; \Delta \vdash^{P} \varphi @ p \text { at } p^{\prime}}{\Gamma ; \Delta \vdash^{P} \varphi \text { at } p} @ E \\
& \frac{\Gamma ; \Delta \vdash^{P} \varphi \text { at } p}{\Gamma ; \Delta \vdash^{P} \diamond \varphi \text { at } p^{\prime}} \diamond I \quad \frac{\Gamma ; \Delta \vdash^{P} \diamond \varphi \text { at } p^{\prime} \quad \Gamma ; \Delta, \varphi \text { at } q \vdash^{P+q} \psi \text { at } p^{\prime \prime}}{\Gamma ; \Delta \vdash^{P} \psi \text { at } p^{\prime \prime}} \diamond E \\
& \frac{\Gamma ; \Delta \vdash^{P+q} \varphi \text { at } q}{\Gamma ; \Delta \vdash^{P} \square \varphi \text { at } p} \square I \quad \frac{\Gamma ; \Delta \vdash^{P} \square \varphi \text { at } p \quad \Gamma, \varphi ; \Delta \vdash^{P} \psi \text { at } p^{\prime}}{\Gamma ; \Delta \vdash^{P} \psi \text { at } p^{\prime}} \square E
\end{aligned}
$$

Figure 1: Natural deduction.

- The local context $\Delta$ is a (possibly empty) finite set of sentences; since a sentence is a pure formula associated to a place. $\Delta$ represents what we assume to be valid in specific places.

- The sentence $\varphi$ at $p$ says that $\varphi$ is derived to be valid in the place $p$ by assuming $\Gamma ; \Delta$.

- The set of places $P$ represents the part of the system we are focusing on.

In the judgement, it is assumed that the places mentioned in $\Gamma$ and $\Delta$ are drawn from the set $P$. More formally, if $\mathrm{PL}(X)$ denotes the set of places that appear in a syntactic object $X$, then it must be the case that $\operatorname{PL}(\Gamma) \cup \operatorname{PL}(\Delta) \cup \operatorname{PL}(\varphi$ at $p) \subseteq P$. Any judgement not satisfying this condition is assumed to be undefined.

A natural deduction system without disjunctive connectives is given in $[17,18]$. The natural deduction system with disjunctive connectives is given in Fig. 1. The most interesting rules are $\diamond E$, 
the elimination of $\diamond$, and $\square I$, the introduction of $\square$. In these rules, $P+p$ denotes the disjoint union $P \cup\{p\}$ and witnesses the fact that the place $p$ occurs in neither $\Gamma$, nor $\Delta$, nor $\varphi$, nor $\psi$. If $p \in P$, then $P+p$ is undefined, and any judgement containing such notation is assumed to be undefined in order to avoid a side condition explicitly stating this requirement.

The rule $\diamond E$ explains how we can use formulae valid at some unspecified location: we introduce a new place and extend the local context by assuming that the formula is valid there. If any assertion that does not mention the new place is validated thus, then it is also validated using the old local context. The rule $\square I$ says that if a formula is validated in some new place without any local assumption on that new place, then that formula must be valid everywhere.

The rules $\diamond I$ and $\square E$ are reminiscent of the introduction of the existential quantification, and the elimination of universal quantification in first-order intuitionistic logic. This analogy, however, has to be taken carefully. For example, if $\Gamma ; \Delta \vdash^{P} \diamond \psi$ at $p$, then we can show using the rules of the logic that $\Gamma ; \Delta \vdash^{P} \square \diamond \psi$ at $p$. In other words, if a formula $\psi$ is true in some unspecified place then every place can deduce that there is some place where $\psi$ is true.

Also note that, as stated, the rule $\perp E$ has a 'local' flavour: from $\perp$ at $p$, we can infer any other property in the same place, $p$. However, the rule has a 'global' consequence. If we have $\perp$ at $p$, then we can infer $\perp @ q \boldsymbol{a t} p$. Using @E, we can then infer $\perp \boldsymbol{a t} q$. Hence, if a set of assumptions makes a place inconsistent, then it will make all places inconsistent.

As we shall see in $\S 2.1$, the Kripke semantics of this logic would be similar to the one given for intuitionistic system IS5 [24, 30, 36]. Hence this logic can be seen as an instance of Hybrid IS5 [7]. Before we proceed to define the Kripke semantics, we illustrate our derivation system by a couple of examples. First example will demonstrate the use of rule $\diamond I$, while the second example will demonstrate the use of $\square E$.

Example 1 Let $p, p^{\prime} \in P, \psi$ be the formula $\left(\operatorname{doc}_{2} \wedge\left(\operatorname{doc}_{2} \rightarrow \operatorname{doc}_{2} @ p\right)\right)$ at $p^{\prime}$. Let $\Delta \stackrel{\text { def }}{=} \diamond \psi$. Pick $q \notin P$ and let $\Delta^{\prime} \stackrel{\text { def }}{=} \diamond \psi, \psi$ at $q$. We can derive

$$
; \Delta \vdash^{P} \operatorname{doc}_{2} \text { at } p
$$

as follows:

$$
\frac{\sum_{\Delta \vdash^{P} \diamond \psi \boldsymbol{a t} p^{\prime}} L \quad ; \Delta^{\prime} \vdash^{P+q} \operatorname{doc}_{2} \text { at } p}{; \Delta \vdash^{P} \operatorname{doc}_{2} \text { at } p} \diamond E
$$

where $\pi$ is the derivation:

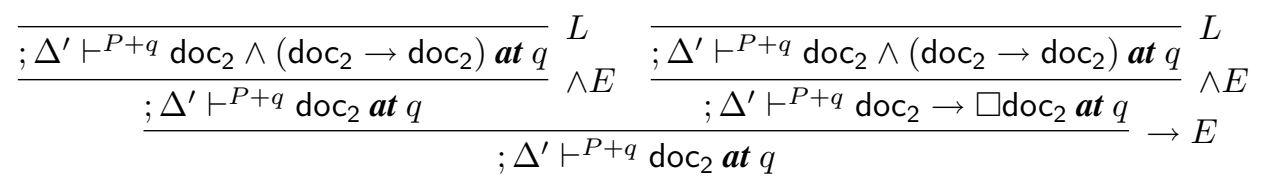

Example 2 Let $p, p^{\prime} \in P$ and $\psi$ be the formula $\left(\operatorname{doc}_{2} \wedge\left(\operatorname{doc}_{2} \rightarrow \square \operatorname{doc}_{2} @ p\right)\right)$ at $p^{\prime}$. Let $\Delta \stackrel{\text { def }}{=} \diamond \psi$. Pick $q \notin P$ and let $\Delta^{\prime} \stackrel{\text { def }}{=} \diamond \psi, \psi$ at $q$. Just as in Example 1, we can derive

$$
; \Delta \vdash^{P} \operatorname{doc}_{2} \text { at } p
$$

as follows: 


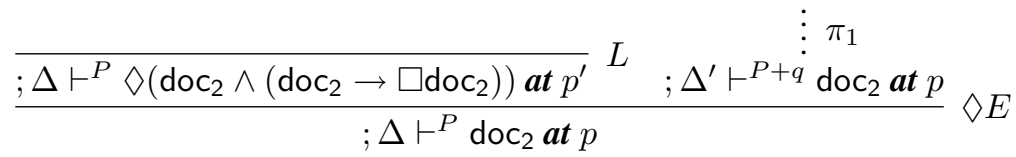

where $\pi_{1}$ is the derivation

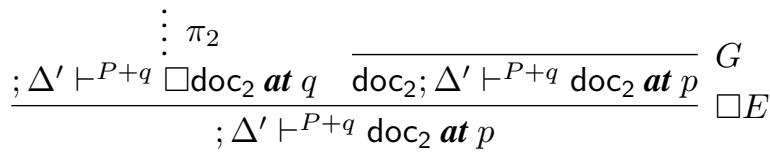

where $\pi_{2}$ is similar to the proof $\pi$ in Example 1 .

\subsection{Kripke Semantics}

There are a number of semantics for intuitionistic logic and intuitionistic modal logics that allow for a completeness theorem [7, 19, 36, 12, 35, 24, 27]. In this section, we concentrate on the semantics introduced by Kripke [20, 37], as it is convenient for applications and fairly simple. This would provide a formalisation of the intuitive concepts introduced above.

In Kripke semantics for intuitionistic propositional logic, logical assertions are interpreted over Kripke models. The validity of an assertion depends on its behaviour as the truth values of its atoms change from false to true according to a Kripke model. A Kripke model consists of a partially ordered set of Kripke states, and an interpretation, I, that maps atoms into states. The interpretation tells which atoms are true in a state. It is required that if an atom is true in a state, then it must remain true in all larger states. Hence, in a larger state more atoms may become true. Consider a logical assertion built from the atoms $A_{1}, \ldots, A_{n}$. The assertion is said to be valid in a state if it continues to remain valid in all larger states.

In order to express the full power of the logic introduced above, we need to enrich the model by introducing places. We achieve this by associating a set of places $P_{k}$ to each Kripke state $k$. The formulae of the logic are validated in these places. The interpretation is indexed by the Kripke states, and the interpretation $I_{k}$ maps atoms into the set $P_{k}$. Since we consider atoms to be resources, the map $I_{k}$ tells how resources are distributed in the Kripke state $k$.

In the case of intuitionistic propositional logic, an atom validated in a Kripke state is validated in all larger states. In order to achieve the corresponding thing, we shall require that all places appearing in a Kripke state appear in every larger state. Furthermore, we require that if $I_{k}$ maps an atom into a place, then $I_{l}$ should map the atom in the same place for all states $l$ larger than $k$. In terms of resources, it means that places in larger states have possibly more resources.

The Kripke models that we shall define now are similar to those defined for the intuitionistic modal system IS5 [12, 35, 24, 27, 7, 36]. In the definition, $K$ is the set of Kripke states, and its elements are denoted by $k, l, \ldots$ The relation $\leq$ is the partial order on the set of states.

Definition 3 (Kripke Model) A quadruple $\mathcal{K}=\left(K, \leq,\left\{P_{k}\right\}_{k \in K},\left\{I_{k}\right\}_{k \in K}\right)$ is a Kripke model if

- $K$ is a (non empty) set;

- $\leq$ is a partial order on $K$;

- $P_{k}$ is a non-empty set of places for all $k \in K$; 
- $P_{k} \subseteq P_{l}$ if $k \leq l$;

- $I_{k}:$ Atoms $\rightarrow \operatorname{Pow}\left(P_{k}\right)$ is such that $I_{k}(A) \subseteq I_{l}(A)$ for all $k \leq l$.

Let $P l s=\bigcup_{k \in K} P_{k}$. We shall say that $P l s$ is the set of places of $\mathcal{K}$.

The definition tells only how resources, i.e. atoms, are distributed in the system. To give semantics to the whole set of formulae $F r m(P l s)$, we need to extend $I_{k}$. The interpretation of a formula depends on its composite parts, and if it is valid in a place in a given state then it remains valid at the same place in all larger states. For example, the formula $\varphi \wedge \psi$ is valid in a state $k$ at place $p \in P_{k}$ if both $\varphi$ and $\psi$ are true at place $p$ in all states $l \geq k$.

The introduction of places in the model allows the interpretation of the spatial modalities of the logic. Formula $\varphi @ p$ is satisfied at a place in a state $k$, if it is true at $p$ in all states $l \geq k ; \diamond \varphi$ and $\square \varphi$ are satisfied at a place in state $k$ if $\varphi$ is true respectively at some or at every place in all states $l \geq k$.

We extend now the interpretation of atoms to interpretation of formulae by using induction on the structure of the formulae. The interpretation of formulae is similar to that used for modal intuitionistic logic $[12,35,24,27,7,36]$.

Definition 4 (Semantics) Let $\mathcal{K}=\left(K, \leq,\left\{P_{k}\right\}_{k \in K},\left\{I_{k}\right\}_{k \in K}\right)$ be a Kripke model with set of places Pls. Given $k \in K, p \in P_{k}$, and a pure formula $\varphi$ with $\mathrm{PL}(\varphi) \subseteq P l s$, we define $(k, p) \models \varphi$ inductively as:

$$
\begin{array}{llll}
(k, p) & =A & \text { iff } & p \in I_{k}(A) ; \\
(k, p) & =T & \text { iff } & p \in P_{k} ; \\
(k, p) \models & & \text { never; } \\
(k, p) \models \varphi \wedge \psi & \text { iff } & (k, p) \models \varphi \text { and }(k, p) \models \psi ; \\
(k, p) \models \varphi \vee \psi & \text { iff } & (k, p) \models \varphi \text { or }(k, p) \models \psi ; \\
(k, p) \models \varphi \rightarrow \psi & \text { iff } & (l \geq k \text { and }(l, p) \models \varphi) \text { implies }(l, p) \models \psi ; \\
(k, p) \models \varphi @ q & \text { iff } & q \in P_{k} \text { and }(k, q) \models \varphi ; \\
(k, p) \models \square & =\text { iff } & \left(l \geq k \text { and } q \in P_{l}\right) \text { implies }(l, q) \models \varphi ; \\
(k, p) & =\Delta \varphi & \text { iff } & \text { there exists } q \in P_{k} \text { such that }(k, q) \models \varphi .
\end{array}
$$

We pronounce $(k, p) \models \varphi$ as '( $k, p)$ forces $\varphi$ ', or ' $(k, p)$ satisfies $\varphi$ '. We write $k \models \varphi$ at $p$ if $(k, p) \models \varphi$.

It is clear from the definition that if $k=\varphi$ at $p$, then $\operatorname{PL}(\varphi$ at $p) \subseteq P_{k}$. Please note that except for logical implication and the modality $\square$, we have not considered larger states in order to interpret a modality or a connective. It turns out that the satisfaction of a formula in a state implies the satisfaction in all larger states, namely if $l \geq k$ then $(k, p) \models \varphi$ implies $(l, p) \models \varphi$ (this is the usual Kripke monotonicity).

Consider the distributed database described when we introduced the logic. We can express the same properties inferred there by using a Kripke model. Fix a Kripke state $k$. The assumption that the two parts, doc ${ }_{1}, \mathrm{doc}_{2}$, can be combined in $p$ in a state $k$ to give the document doc can be expressed as $(k, p)=\left(\operatorname{doc}_{1} \wedge \mathrm{doc}_{2}\right) \rightarrow$ doc. If the resources doc $c_{1}$ and $\mathrm{doc}_{2}$ are assigned to the place $p$, i.e., $(k, p) \models \operatorname{doc}_{1}$ and $(k, p) \models \operatorname{doc}_{2}$, then, since $(k, p) \models \operatorname{doc}_{1} \wedge \operatorname{doc}_{2}$, it follows that $(k, p) \models$ doc.

Let us consider a slightly more complex situation. Suppose that $k \models \diamond\left(\operatorname{doc}_{2} \wedge\left(\operatorname{doc}_{2} \rightarrow\right.\right.$ $\left.\left.\square \mathrm{doc}_{2}\right)\right)$ at $p^{\prime}$. According to the semantics of $\diamond$, there is some place $r$ such that $(k, r) \models \operatorname{doc}_{2} \wedge$ $\left(\operatorname{doc}_{2} \rightarrow \square \mathrm{doc}_{2}\right)$. The semantics of $\wedge$ tells us that $(k, r) \models \operatorname{doc}_{2}$ and $(k, r) \models\left(\operatorname{doc}_{2} \rightarrow \square \mathrm{doc}_{2}\right)$. 
We can conclude from the semantics of $\rightarrow$ that $(k, r) \models \square$ doc $_{2}$, and the semantics of $\square$ then gives us that $(k, p) \models \operatorname{doc}_{2}$. Therefore, if $\operatorname{doc}_{1}$ is placed at $p$ in the state $k$, then the whole document doc would become available at place $p$ in state $k$.

To give semantics to the judgements of the logic, we need to extend the definition of forcing relation to judgements. We begin by extending the definition to contexts.

Definition 5 (Forcing on Contexts) Let $\mathcal{K}=\left(K, \leq,\left\{P_{k}\right\}_{k \in K},\left\{I_{k}\right\}_{k \in K}\right)$ be a Kripke model. Given a state $k$ in $K$, a finite set of pure formulae $\Gamma$, and a finite set of sentences $\Delta$ such that $\operatorname{PL}(\Gamma ; \Delta) \subseteq$ $P_{k}$; we say that $k$ forces the context $\Gamma ; \Delta$ (and we write $k \models \Gamma ; \Delta$ ) if

1. for every $\varphi \in \Gamma$ and every $p \in P_{k}:(k, p) \models \square \varphi$;

2. for every $\psi \boldsymbol{a t} q \in \Delta:(k, q) \models \psi$.

Finally, we extend the definition of forcing to judgements.

Definition 6 (Judgment Satisfaction) Let $\mathcal{K}=\left(K, \leq,\left\{P_{k}\right\}_{k \in K},\left\{I_{k}\right\}_{k \in K}\right)$ be a Kripke model. The judgement $\Gamma ; \Delta \vdash^{P} \mu$ at $p$ is said to be valid in $\mathcal{K}$ if

- $\operatorname{PL}(\Gamma) \cup \operatorname{PL}(\Delta) \cup \operatorname{PL}(\mu) \cup\{p\} \subseteq P$;

- for every $k \in K$ such that $P \subseteq P_{k}$, if $k \models \Gamma ; \Delta$ then $(k, p) \models \mu$.

Moreover, we say that $\Gamma ; \Delta \vdash^{P} \mu$ at $p$ is valid (and we write $\Gamma ; \Delta \models \mu$ at $p$ ) if it is valid in every Kripke model.

Although, it is possible to obtain soundness and completeness of Kripke semantics directly, we shall not do so in this paper. Instead, they will be derived as corollaries. Soundness will follow from the soundness of birelational semantics and encoding of Kripke models into birelational models. Completeness will emerge as a corollary in the proof of construction of finite counter-model.

\section{Birelational Models}

One other semantics given for modal intuitionistic logics in literature is birelational semantics [12, $35,27,36]$. As in the case of intuitionistic modal logics [25, 36], birelational semantics for our logic enjoys the finite model property, while Kripke semantics does not.

Birelational models, like Kripke models, have a set of partially ordered states. The partially ordered states will be called worlds, and we use $u, v, w, \ldots$ to range over them. Formulae will be validated in worlds, and if a formula is validated in a world, then it will be validated in all larger worlds. To validate atoms we have the interpretation $I$, which maps atoms into a subset of worlds. If $I$ maps an atom into a world, then it will map the atom in all larger worlds.

In addition to the partial order, however, there is also a second binary relation on the set of states which is called reachability or accessibility relation. Intuitively, $u R w$ means that $w$ will be reachable from $u$. As our logic is a hybridisation for $I S 5$, the relation $R$ will be an equivalence relation. The relation $R$ will also satisfy a technical requirement, the reachability condition, that is necessary to ensure monotonicity and soundness of the logic.

Unlike the Kripke semantics, the states will not have a set of places associated to them. Instead, there is a partial function, Eval, which maps a world to a single place. In a sense which we will make precise in $\S 3.2$, a world in a birelational model corresponds to a place in a specific Kripke 
state. As we shall see later, the partiality of the function Eval is crucial in the proof of the finite model property. In the case $\operatorname{Eval}(w)$ is defined and is $p$, we shall say that $w$ evaluates to $p$.

In addition to partiality, Eval will also satisfy two other properties: coherence and uniqueness. Coherence says that if a world evaluates to $p$, then all larger worlds evaluate to $p$. Together with the reachability condition, coherence will ensure the monotonicity of the modality @. Uniqueness will say that no two worlds reachable from each other can evaluate to the same place. Uniqueness will be essential for the soundness of introduction of conjunction $(\wedge I)$ and implication $(\rightarrow I)$. The formal definition of the models is below.

Definition 7 (Birelational Model) Given a set of places Pls, a birelational model on Pls is a quintuple $\mathcal{W}_{P l s}=(W, \leq, R, I$, Eval $)$, where

1. $W$ is a (non empty) set, ranged over by $v, v^{\prime}, w, w^{\prime}, \ldots$

2. $\leq$ is a partial order on $W$.

3. $R \subseteq W \times W$ is an equivalence relation and satisfies the reachability condition:

$$
\text { if } w^{\prime} \geq w R v \text { then there exists } v^{\prime} \text { such that } w^{\prime} R v^{\prime} \geq v
$$

4. $I:$ Atoms $\rightarrow \operatorname{Pow}(W)$ is such that if $w \in I(A)$ then $w^{\prime} \in I(A)$ for all $w^{\prime} \geq w$.

5. Eval $: W \rightarrow P l s$ is a partial function. We write $v \uparrow$ if $\operatorname{Eval}(v)$ is not defined, $v \downarrow$ if $\operatorname{Eval}(v)$ is defined, and $v \downarrow p$ if $\operatorname{Eval}(v)$ is defined and equal to $p$.

Moreover, the following properties hold:

(a) coherence: for any $v \in W$, if $v \downarrow p$ then $w \downarrow p$ for every $w \geq v$;

(b) uniqueness: for every $v \in W$ such that $v \downarrow p$, if $v R v^{\prime}$ and $v^{\prime} \downarrow p$, then $v=v^{\prime}$.

In addition to the reachability condition, usually there is another similar condition in birelational models for intuitionistic modal logics [12, 35, 27, 36]:

$$
\text { if } w R v \leq v^{\prime} \text { then there exists } w^{\prime} \text { such that } w \leq w^{\prime} R v^{\prime}
$$

In this case, as $R$ is an equivalence relation, the property is an immediate consequence of the reachability condition.

As in the case of Kripke models, the interpretation of atoms extends to formulae. A formula $\varphi @ p$ is true in a world $w$, if there is a reachable world which evaluates to $p$ and where $\varphi$ is valid. A formula $\nabla \varphi$ is valid in a world $w$, if there is a reachable world (not necessary in the domain of Eval) where $\varphi$ is valid. A formula $\square \varphi$ is valid in a world $w$ if $\varphi$ is valid in all worlds reachable from worlds $w^{\prime}$ larger than $w$.

Definition 8 (Bi-forcing Semantics) Let $\mathcal{W}_{P l s}=(W, \leq, R, I$, Eval $)$ be a birelational model on Pls. Given $w \in W$, and a pure formula $\varphi \in \operatorname{Frm}(P l s)$, we define the forcing relation $w \models \varphi$ 
inductively as follows:

$$
\begin{array}{llll}
w \models A & \text { iff } \quad & w \in I(A) ; \\
w \models T & & \text { for all } w \in W ; \\
w \models \perp & & \text { never; } \\
w \models \varphi \wedge \psi & \text { iff } & w \models \varphi \text { and } w \models \psi ; \\
w \models \varphi \vee \psi & \text { iff } & w \models \varphi \text { or } w \models \psi ; \\
w \models \varphi \rightarrow \psi & \text { iff } & (v \geq w \text { and } v \models \varphi) \text { implies } v \models \psi ; \\
w \models \varphi @ q & \text { iff } & \text { there exists } v \text { such that } w R v, v \downarrow q \text { and } v \models \varphi ; \\
w \models \square \varphi & \text { iff } & \left(v \geq w \text { and } v R v^{\prime}\right) \text { implies } v^{\prime} \models \varphi ; \\
w \models \nabla \varphi & \text { iff } & \text { there exists } v \in W \text { such that } w R v \text { and } v \models \varphi .
\end{array}
$$

We pronounce $w \models \varphi$ as ' $w$ forces $\varphi$,' or ' $w$ satisfies $\varphi$.'

As in the case for Kripke models, this relation is monotone: if $v \geq w$ then $w=\varphi$ implies $v=\varphi$.

Example 9 Consider the birelational model $\mathcal{W}_{\text {exam }}$ with two worlds, say $w_{1}$ and $w_{2}$. Let $w_{1}$ and $w_{2}$ be reachable from each other and let $w_{1} \leq w_{2}$. The world $w_{2}$ evaluates to $p$, while the evaluation of $w_{1}$ is undefined. Let $A$ be an atom. We define $I(A)$ to be the singleton $\left\{w_{2}\right\}$. For any formula $\varphi$, we abbreviate $\varphi \rightarrow \perp$ as $\neg \varphi$.

Consider the pure formula $\neg A$. Now, by definition, $w_{2} \models A$ and therefore $w_{2} \not \models \neg A$. Also, as $w_{1} \leq w_{2}$, we get $w_{1} \not \models \neg A$. This means that $w_{2} \models \neg \neg A$, and $w_{1} \models \neg \neg A$. Hence, we get $w_{1}, w_{2} \models \square \neg \neg A$.

On the other hand, consider the formula $\neg \neg \square A$. We have by definition that $w_{1} \not \models A$. As $w_{1}$ is reachable from both $w_{1}$ and $w_{2}$, we deduce that $w_{1}, w_{2} \not \models \square A$. Using the semantics of $\rightarrow$, we get that $w_{1}, w_{2} \not \models \neg \neg \square A$.

We now extend the semantics to the judgements of the logic. We begin by extending the semantics to contexts.

Definition 10 (Bi-forcing on Contexts) Let $\mathcal{W}_{P l s}=(W, \leq, R, I$, Eval) be a birelational model on Pls. Given a finite set of pure formulae $\Gamma$, and a finite set of sentences $\Delta$, such that $\operatorname{PL}(\Gamma ; \Delta) \subseteq$ $P l s$; we say that $w \in W$ forces the context $\Gamma ; \Delta$ (and we write $w \models \Gamma ; \Delta$ ) if

1. for every $\varphi \in \Gamma: w \models \square \varphi$, and

2. for every $\psi$ at $q \in \Delta: w \models \psi @ q$.

In order to extend the semantics to judgements, we need one more definition. We say that a place $p$ is reachable from a world $v$ if there is a world which evaluates to $p$ and is reachable from $v$. The set of all places reachable from a world $v$ will be denoted by Reach $(v)$. More formally,

$$
\operatorname{Reach}(v) \stackrel{\text { def }}{=}\{p: w \downarrow p \text { for some } w \in W, v R w\}
$$

It can be easily shown by using the reachability condition and coherence that if $v \leq w$, then every place reachable from $v$ is also reachable from $w$, namely $\operatorname{Reach}(v) \subseteq \operatorname{Reach}(w)$. Moreover, if $v R w$, then $\operatorname{Reach}(v)=\operatorname{Reach}(w)$. We now extend the definition of satisfaction to judgements.

Definition 11 (Bi-satisfaction for Judgments) The sequent $\Gamma ; \Delta \vdash^{P} \varphi$ at $p$ is said to be valid in the birelational model $\mathcal{W}_{P l s}=(W, \leq, R, I, E v a l)$ if: 
- $P L(\Gamma) \cup P L(\Delta) \cup\{p\} \subseteq P$;

- for any $w \in W$ such that $P \subseteq \operatorname{Reach}(w): w \models \Gamma ; \Delta$ implies $w \models \varphi @ p$.

Moreover, we say that $\Gamma ; \Delta \vdash^{P} \mu$ at $p$ is bi-valid (and we write $\Gamma ; \Delta \models{ }^{P} \mu$ at $p$ ) if it is valid in every birelational model.

Example 12 Consider the birelational model $\mathcal{W}_{\text {exam }}$ on two worlds $w_{1}$ and $w_{2}$ discussed in Example 9. We had $w_{1}, w_{2} \models \square \neg \neg A$ and $w_{1}, w_{2} \not \models \neg \neg \square A$. Therefore, the judgement $; \vdash\{p\} \square \neg \neg A$ at $p$ is bi-valid in the model $\mathcal{W}_{\text {exam }}$, while the judgement $\square \neg \neg A$ at $p \vdash\{p\} \neg \neg \square A$ at $p$ is not bi-valid in $\mathcal{W}_{\text {exam }}$.

In fact, we will later on show that the judgement $; \square \neg \neg A$ at $p \vdash\{p\} \neg \neg \square A$ at $p$ is valid in every finite Kripke model. Therefore this example, adapted from [25, 36], will demonstrate that the finite model property does not hold in the case of Kripke semantics.

\subsection{Soundness}

The proof of soundness has several subtleties, that arise as a consequence of the inference rules for the introduction of $\square$ ( $\square \mathrm{I})$ and elimination of $\diamond(\diamond \mathrm{E})$. Let us illustrate this for the case of $\square I$. Recall the inference rule of $\square I$ from Fig. 1:

$$
\frac{\Gamma ; \Delta \vdash^{P+q} \varphi \text { at } q}{\Gamma ; \Delta \vdash^{P} \square \varphi \text { at } p} \square I
$$

To show the soundness of this rule, we must show that the judgement $\Gamma ; \Delta \vdash^{P} \square \varphi$ at $p$ is bivalid whenever the judgement $\Gamma ; \Delta \vdash^{P+q} \varphi$ at $q$ is bi-valid. In order to show that the judgement $\Gamma ; \Delta \vdash^{P} \square \varphi$ at $p$ is bi-valid, we must consider an arbitrary world, say $w$, in an arbitrary birelational model, say $\mathcal{W}_{P l s}$, such that $P \subseteq \operatorname{Reach}(w)$ and $w \models \Gamma ; \Delta$. We need to prove that $w \models \square \varphi @ p$ also. For this, we need to show that for any world $v$ in $\mathcal{W}_{P l s}$ such that $w \leq w^{\prime} R v$ for some $w^{\prime}$, it is the case that $v=\varphi$. Pick one such $v$ and fix it.

Please note that without loss of generality, we can assume that Pls does not contain $q$ (otherwise, we can always rename $q$ in the model). To use the hypothesis that $\Gamma ; \Delta \vdash^{P+q} \varphi$ at $q$ is bi-valid, we must consider a modification of $\mathcal{W}_{P l s}$. One strategy, that is adopted in the case of Kripke semantics [7], is to add new worlds $v_{q}^{\prime}$, one for each world $v^{\prime} \geq v$. The new worlds $v_{q}^{\prime}$ duplicate $v^{\prime}$ in all respects except that they evaluate to $q$. If the resulting construction yields a birelational model, then $\operatorname{Reach}\left(v_{q}^{\prime}\right)$ would contain $P$ as well as $q$.

The next step would be to show that any formula $\psi$, that does not refer to the place $q$, is satisfied by $v_{q}^{\prime}$ if and only if it is satisfied by $v^{\prime}$. Using this, we get that $v_{q}^{\prime}$ forces the context $\Gamma ; \Delta$ in the new model also. Then, we can use the hypothesis to obtain that $v_{q}^{\prime}$ satisfies $\varphi @ q$. Since $v_{q}^{\prime}$ evaluates to $q$, we will get that $v_{q}^{\prime}$ forces $\varphi$. As $\varphi$ does not refer to $q$, we will get that $v^{\prime}$ forces $\varphi$. We can then conclude the proof by observing that $v \geq v$, and choosing $v^{\prime}$ to be $v$.

In fact, if the world $v$ was in the domain of Eval, then the above outline would have worked. However, this breaks down in case $v \uparrow$. To illustrate this, suppose that there is a world $v^{\prime}$ such that $v \leq v^{\prime}, v^{\prime} \uparrow$ and $v R v^{\prime}$. In the construction of the extension, we would thus have two worlds $v_{q}$ and $v_{q}^{\prime}$ reachable from each other that evaluate to the same place $q$. This violates the uniqueness condition.

This breakdown is fatal for the proof and cannot be fixed. Coherence demands that $v_{q}^{\prime} \downarrow q$ if $v_{q} \downarrow q$. So, we cannot fiddle with the evaluation. We cannot even relax uniqueness as this will be needed 
for soundness of introduction of conjunction $(\wedge I)$ and implication $(\rightarrow I)$. Furthermore, we cannot require that the evaluation is a total function: it is the partiality of this function that gives us the finite model property. Indeed, if the function was total, the class of birelational models would be equivalent to the class of Kripke models, and we would have not gained anything by using birelational models.

Our strategy to prove soundness is to construct a birelational model from $\mathcal{W}_{P l s}$, called $q$-extension, whose worlds are the union of two sets. The first one of these sets is the reachability relation $R$ of $\mathcal{W}_{P l s}$. The second one will be the Cartesian product $\{q\} \times W$, where $W$ is the set of worlds of $\mathcal{W}_{\text {Pls }}$. Hence, the worlds of the $q$-extension are ordered pairs. A world $\left(w^{\prime}, w\right)$ will evaluate to the same place as $w^{\prime}$, and $(q, w)$ will evaluate to $q$. Two worlds will be reachable from each other only if they agree in the second entry.

The construction would guarantee (see Lemma 14) that given $\psi \in \operatorname{Frm}(P l s)$, the world $\left(w^{\prime}, w\right)$ satisfies $\psi$ if and only if $w^{\prime}$ does, and the world $(q, w)$ satisfies $\psi$ if and only if $w$ does. The proof of soundness of $\square I$ would work as follows. Let $v$ be the fixed world as above. Consider the world $(q, v)$ in the $q$-extension. We will show that $v$ satisfies $\Gamma ; \Delta$, and hence $(q, v)$ satisfies $\Gamma ; \Delta$. The set of reachable places from $(q, v)$ contains $P$ as well as $q$, and we can thus conclude that $(q, v)$ satisfies $\varphi @ q$. Since $(q, v)$ evaluates to $q$, we conclude that $(q, v)$ satisfies $\varphi$. As mentioned above, this is equivalent to saying that $v$ satisfies $\varphi$.

We are ready to carry out this proof formally. We begin by constructing the $q$-extension, and showing that this is a birelational model.

Lemma 13 ( $q$-Extension) Let $\mathcal{W}_{P l s}=(W, \leq, R, I$, Eval $)$ be a birelational model on Pls. Given a new place $q \notin P l s$, we define the $q$-extension $\mathcal{W}\langle q\rangle_{P l s^{\prime}}$ to be the quintuple $\left(W^{\prime}, \leq^{\prime}, R^{\prime}, I^{\prime}, E v a l^{\prime}\right)$, where

1. $P l s \stackrel{\text { def }}{=} P l s \cup\{q\}$.

2. $W^{\prime} \stackrel{\text { def }}{=} R \cup(\{q\} \times W)$.

3. $\leq^{\prime} \subseteq W^{\prime} \times W^{\prime}$ is defined as:

- $\left(w^{\prime}, w\right) \leq \prime\left(v^{\prime}, v\right)$ if and only if $w^{\prime} \leq v^{\prime}$ and $w \leq v$,

- $(q, w) \leq^{\prime}(q, v)$ if and only if $w \leq v$;

4. $R^{\prime} \subseteq W^{\prime} \times W^{\prime}$ is defined as:

- $\left(w^{\prime}, w\right) R^{\prime}\left(v^{\prime}, w\right)$,

- $\left(w^{\prime}, w\right) R^{\prime}(q, w)$,

- $(q, w) R^{\prime}\left(w^{\prime}, w\right)$, and

- $(q, w) R^{\prime}(q, w)$.

5. $I^{\prime}:$ Atoms $\rightarrow \operatorname{Pow}\left(W^{\prime}\right)$ is defined as:

- $I^{\prime}(A) \stackrel{\text { def }}{=}\left\{\left(w^{\prime}, w\right) \mid w^{\prime} \in I(A), w^{\prime} R w\right\} \cup\{(q, w) \mid w \in I(A)\} ;$

6. Eval ${ }^{\prime}: W^{\prime} \rightarrow P l s^{\prime}$ is defined as

- $\operatorname{Eval}^{\prime}\left(\left(w^{\prime}, w\right)\right) \stackrel{\text { def }}{=} \operatorname{Eval}\left(w^{\prime}\right)$ for every $\left(w^{\prime}, w\right) \in R,{ }^{1}$

${ }^{1}$ In the equality, the left hand side is defined only if the right hand side is. 


$$
\text { - } \operatorname{Eval}^{\prime}((q, w)) \stackrel{\text { def }}{=} q \text { for every } w \in W \text {. }
$$

The $q$-extension is a birelational model.

Proof We need to show the five properties of Definition 7. As an example, we prove here uniqueness property: two different worlds reachable from each other cannot evaluate to the same place. As $(q, v)$ always evaluates to $q$, two worlds $(w, v)$ and $(q, w)$ cannot evaluate to the same place. There are two other possible cases.

Case a. Suppose $\left(v^{\prime}, v\right) R^{\prime}\left(w^{\prime}, w\right),\left(w^{\prime}, w\right) \downarrow p$ and $\left(v^{\prime}, v\right) \downarrow p$. We have by definition $v^{\prime} R v, w^{\prime} R w$, $v=w, w^{\prime} \downarrow p$ and $v^{\prime} \downarrow p$. Since $R$ is an equivalence and $v=w$, we get $v^{\prime} R w^{\prime}$. By uniqueness on $\mathcal{W}_{P l s}$, we get $v^{\prime}=w^{\prime}$. Therefore $\left(v^{\prime}, v\right)=\left(w^{\prime}, w\right)$.

Case $b$. Suppose that $(q, v) R^{\prime}(q, w),(q, w) \downarrow q$ and $(q, v) \downarrow q$. We have by definition $v=w$, and hence $(q, v)=(q, w)$.

We will now show that if a pure formula, say $\psi$, does not mention $q$ then $\left(w^{\prime}, w\right)$ satisfies $\psi$ only if $w^{\prime}$ does. Furthermore, $(q, w)$ satisfies $\psi$ only if $w$ does.

Lemma $14\left(\mathcal{W}\langle u, q\rangle_{P l s^{\prime}}\right.$ is conservative) Let $\mathcal{W}_{P l s}=(W, \leq, R, I$, Eval $)$ be a birelational model, and let $\mathcal{W}\langle q\rangle_{P l s^{\prime}}=\left(W^{\prime}, \leq^{\prime}, R^{\prime}, I^{\prime}, E v a l^{\prime}\right)$ be its $q$-extension. Let $\models$ and $\models{ }^{\prime}$ extend the interpretation of atoms in $\mathcal{W}_{P l s}$ and $\mathcal{W}\langle q\rangle_{P l s^{\prime}}$ respectively. For every $\varphi \in \operatorname{Frm}(P l s)$ and $w \in W$, the following hold:

1. for every $w^{\prime} R w,\left(w^{\prime}, w\right) \models^{\prime} \varphi$ if and only if $w^{\prime} \models \varphi$; and

2. $(q, w) \models^{\prime} \varphi$ if and only if $w \models \varphi$.

Proof Prove both the points simultaneously by induction on the structure of formulae in $\mathrm{Frm}(\mathrm{Pls})$. The base case of induction is verified on atoms, on $T$, and on $\perp$ by definition. For the inductive step, here we show how to prove the point 1 in the $\square$ case, and for the other cases we refer to the companion technical report [11].

Consider the formula $\square \varphi$, and assume that $\left(w^{\prime}, w\right) \models^{\prime} \square \varphi$. This means that $\varphi$ is forced by every world reachable from some world larger that $\left(w^{\prime}, w\right)$. In particular, we have that

$$
\text { for every }\left(v^{\prime}, v\right) \geq\left(w^{\prime}, w\right) \text {, if }\left(v^{\prime \prime}, v\right) R^{\prime}\left(v^{\prime}, v\right) \text { then }\left(v^{\prime \prime}, v\right) \models^{\prime} \varphi \text {. }
$$

We need to show that $w^{\prime}=\square \varphi$. Pick $v^{\prime}, v^{\prime \prime}$ such that $v^{\prime} \geq w^{\prime}$, and $v^{\prime \prime} R v^{\prime}$, and fix them. It suffices to show that $v^{\prime \prime}=\varphi$.

Since $v^{\prime} \geq w^{\prime}$ and $w^{\prime} R w$, the reachability condition for $R$ says that there exists $v \in W$ such that $v^{\prime} R v \geq w$. By transitivity, we have $v^{\prime \prime} R v$ also. Hence $\left(v^{\prime}, v\right) \geq^{\prime}\left(w^{\prime}, w\right)$ and $\left(v^{\prime \prime}, v\right) R^{\prime}\left(v^{\prime}, v\right)$. Property (2) says that $\left(v^{\prime \prime}, v\right) \models{ }^{\prime} \varphi$, and so $v^{\prime \prime} \models \varphi$ by induction hypothesis.

For the other direction, assume $w^{\prime}=\square \varphi$. Then

$$
\text { for every } v^{\prime} \geq w^{\prime} \text {, if } v^{\prime \prime} R v^{\prime} \text { then } v^{\prime \prime}=\varphi \text {. }
$$

We need to show that $\left(w^{\prime}, w\right) \models \models^{\prime} \square \varphi$.

Consider a world $\left(v^{\prime}, v\right) \geq^{\prime}\left(w^{\prime}, w\right)$, and fix it. We have $v^{\prime} R v, v^{\prime} \geq w^{\prime}$ and $v \geq w$. Now, consider any world reachable from $\left(v^{\prime}, v\right)$. We need to show that this world satisfies $\varphi$. There are two possible cases. If the world is of the form $\left(v^{\prime \prime}, v\right)$, we have that $v^{\prime \prime} R v$. Since $v^{\prime} R v$, we get $v^{\prime \prime} R v^{\prime}$. Since $v^{\prime} \geq w^{\prime}$, we get $v^{\prime \prime} \models \varphi$ by (3). Hence, $\left(v^{\prime \prime}, v\right) \models \models^{\prime} \varphi$, by induction hypothesis. In the other case, the world is of the form $(q, v)$. Since $v R v^{\prime}$ and $v^{\prime} \geq w^{\prime}$, we have $v \models \varphi$ by (3). Therefore, $(q, v) \models^{\prime} \varphi$ by induction hypothesis. 
We are ready to prove soundness, which depends on Lemmas 13 and 14. This theorem provides not only soundness for birelational models, but also for Kripke models, thanks to the encoding presented in $\$ 3.2$. One last remark, easy to check, is that if a world $w$ satisfies a context then any world reachable from and/or greater than it also satisfies the context. Formally

$$
\text { if } w \models \Gamma ; \Delta \text { then } v \models \Gamma ; \Delta \text { for every } v \text { such that } v R w \text { or } v \geq w \text {. }
$$

Theorem 15 (Bi-soundness) If the judgement $\Gamma ; \Delta \vdash^{P} \mu$ at $p$ is derivable in the logic, then it is bi-valid.

Proof The proof proceeds by induction on $n$, the number of inference rules applied in the derivation of the judgement $\Gamma ; \Delta \vdash^{P} \mu$ at $p$. The inference rules are given in Fig. 1. The base case, where only one inference rule is used to derive the judgement, follows easily from the definition. We discuss the induction step.

Induction hypothesis $(n>1)$. We assume that the theorem holds for any judgement that is deducible by applying less than $n$ instances of inference rules, and consider a judgement $\Gamma ; \Delta \vdash^{P}$ $\mu$ at $p$ derivable in the logic by using exactly $n$ instances.

We fix a model $\mathcal{W}_{P l s}=(W, \leq, R, V, E v a l)$ on $P l s$, and let $\models$ be the forcing relation in this model. Let $w \in W$ be such that $P \subseteq \operatorname{Reach}(w)$ and $w \models \Gamma ; \Delta$. Fix $w$ for the rest of the proof. We have to show $w \models \mu @ p$. We proceed by cases by considering the last rule applied to obtain $\Gamma ; \Delta \vdash^{P} \mu$ at $p$. For the sake of clarity, we consider only the cases in which the last rule is introduction of implication $(\rightarrow I)$ and introduction of $\square(\square \mathrm{I})$. The treatment of the other rules is similar.

- Case $\rightarrow I$. If the last inference rule used was $\rightarrow I$ then $\mu$ is of the form $\varphi \rightarrow \psi$, and $\operatorname{PL}(\Gamma ; \Delta) \cup \operatorname{PL}(\varphi) \cup \operatorname{PL}(\psi) \cup\{p\} \subseteq P$. Furthermore, $\Gamma ; \Delta, \varphi$ at $p \vdash^{P} \psi$ at $p$ by using less than $n$ instances of the inference rules. By induction hypothesis, $\Gamma$; $\Delta, \varphi$ at $p \vdash^{P} \psi$ at $p$ is bi-valid. We have to prove that there exists $v R w$ such that $v \downarrow p$ and $v \models \varphi \rightarrow \psi$.

Since $P \subseteq \operatorname{Reach}(w)$, there exists $v \in R(w)$ such that $v \downarrow p$. We will prove that $v \models \varphi \rightarrow \psi$. Pick $v^{\prime} \geq v$ and fix it. We need to show that if $v^{\prime}=\varphi$, then $v^{\prime}=\psi$ also.

We have $v^{\prime} \downarrow p$ by coherence property, and $v^{\prime}=\Gamma ; \Delta$. Also, as $R$ is reflexive, we have $v^{\prime} R v^{\prime}$. If we assume that $v^{\prime}=\varphi$, then we get by definition that $v^{\prime}=\varphi @ p$. Hence, we get $v^{\prime}=\Gamma ; \Delta, \varphi$ at $p$. By induction hypothesis $\Gamma ; \Delta, \varphi$ at $p \vdash^{P} \psi$ at $p$ is bi-valid, and therefore $v^{\prime} \models \psi @ p$.

Therefore, there is a world reachable from $v^{\prime}$ which evaluates to $p$ and which forces $\psi$. Since $v^{\prime} \downarrow p$ and $v^{\prime} R v^{\prime}$, uniqueness says that this world must be $v^{\prime}$ itself. Therefore $v^{\prime} \models \psi$, as required.

- Case $\square I$. Then $\mu$ is of the form $\square \varphi$. Moreover, $\operatorname{PL}(\Gamma ; \Delta) \cup \operatorname{PL}(\varphi) \cup\{p\} \subseteq P$, and $\Gamma ; \Delta \vdash^{P+q} \varphi$ at $q$ for some $q \notin P$ by using less that $n$ instances of the rules. By induction hypothesis, $\Gamma$; $\Delta \vdash^{P+q} \varphi$ at $q$ is bi-valid. Without loss of generality, we can assume that $q \notin P l s$ (otherwise, we can rename $q$ in $P l s$ ).

We have that $w \models \Gamma ; \Delta$, and we need to show that $w \models \square \varphi @ p$. Note that $p \in P$, and $P \subseteq \operatorname{Reach}(w)$. Therefore there is a $w^{\prime} \in \operatorname{Reach}(w)$ such that $w^{\prime} \downarrow p$. Pick such a $w^{\prime}$, and fix it. We have $w^{\prime} \models \Gamma$; $\Delta$. We shall show that $w^{\prime} \models \square \varphi$, and we will be done.

In order to show that $w^{\prime} \models \square \varphi$, we have to show that $v^{\prime} \models \varphi$ for every $v, v^{\prime}$ such that $v^{\prime} R v \geq w$. Pick such $v, v^{\prime}$ and fix them. We have $v^{\prime} \models \Gamma ; \Delta$. Since $P \subseteq \operatorname{Reach}(w)$ and $v^{\prime} R v \geq w$, we get $P \subseteq \operatorname{Reach}\left(v^{\prime}\right)$ by reachability and coherence. 
Let $P l s^{\prime}=P l s \cup\{q\}$, and let $\mathcal{W}\langle q\rangle_{P l s^{\prime}}$ be the $q$-extension of the birelational model. Let $\models^{\prime}$ be the forcing relation on $\mathcal{W}\langle u, q\rangle$. From the hypothesis $v^{\prime}=\Gamma ; \Delta$ and Lemma 14, we get $\left(v^{\prime}, v^{\prime}\right) \models{ }^{\prime} \Gamma ; \Delta$.

From definition of $q$-extension, it is clear that Reach $\left(\left(v^{\prime}, v^{\prime}\right)\right)=\operatorname{Reach}\left(v^{\prime}\right) \cup\{q\}$. Hence $P+q \subseteq \operatorname{Reach}\left(\left(v^{\prime}, v^{\prime}\right)\right)$. We can now apply the induction hypothesis on the world $\left(v^{\prime}, v^{\prime}\right)$ and obtain $\left(v^{\prime}, v^{\prime}\right) \models^{\prime} \varphi @ q$. By the definition of the $q$-extension, this is equivalent to $\left(q, v^{\prime}\right) \models^{\prime} \varphi$. Lemma 14 then implies that $v^{\prime} \models \varphi$, as required.

\subsection{Relating Kripke and Birelational Models}

In this section, we shall present an encoding of Kripke models in birelational models that preserves the forcing relation. This will allow us to prove the soundness of the logic for Kripke models.

In particular, given a Kripke model with a set of states $K$, we construct a birelational model whose worlds are pairs $(k, p)$ where $k \in K$ and $p$ is a place in the Kripke state $k$. Two worlds will be related if they come from the same Kripke state. The world $(l, p)$ will be greater that $(k, q)$ only if $l \geq k$ and $p=q$. The world $(k, p)$ will evaluate to $p$, and an atom will be interpreted in the world $(k, p)$ only if it is placed in $p$ in the Kripke state $k$. The construction will guarantee that the Kripke state $k$ forces an assertion $\psi @ p$ if and only if the corresponding world $(k, p)$ forces the formula $\psi$.

Proposition 16 (Encoding) Given a Kripke model, $\mathcal{K}=\left(K, \leq,\left\{P_{k}\right\}_{k \in K},\left\{I_{k}\right\}_{k \in K}\right)$ with set of places $P l s$, we define its $\mathcal{K}$-birelational model $\mathcal{W}_{P l s}^{\mathcal{K}}$ to be the quintuple $\left(W^{\prime}, \leq^{\prime}, R^{\prime}, I^{\prime}, E v a l^{\prime}\right)$, where

1. $W^{\prime} \stackrel{\text { def }}{=} \bigcup_{k \in K}\left\{(k, p): p \in P_{k}\right\}$;

2. $\leq^{\prime} \subseteq W^{\prime} \times W^{\prime}$ is defined as: $(k, p) \leq^{\prime}(l, q)$ if and only if $k \leq l$ and $p=q$;

3. $R^{\prime}: \subseteq W^{\prime} \times W^{\prime}$ is defined as: $(k, p) R^{\prime}(l, q)$ if and only if $k=l$;

4. $I^{\prime}:$ Atoms $\rightarrow \operatorname{Pow}\left(W^{\prime}\right)$ is defined as: $I(A) \stackrel{\text { def }}{=}\left\{(k, p) \mid p \in I_{k}(A)\right\}$;

5. $E_{v a l}^{\prime}: W^{\prime} \rightarrow P l s^{\prime}$ is defined as: $\operatorname{Eval}(k, p) \stackrel{\text { def }}{=} p$.

$\mathcal{W}_{P l s}^{\mathcal{K}}$ is a birelational model. Let $\left.\right|_{\mathcal{K}}$ and $=_{\mathcal{W}}$ extend the interpretation of atoms in $\mathcal{K}$ and $\mathcal{W}_{P l s}^{\mathcal{K}}$ respectively. The encoding preserves the forcing relation, namely for every $k \in K, p \in P_{k}$, and $\varphi \in$ $\operatorname{Frm}(P l s)$ :

$$
(k, p) \models_{\mathcal{K}} \varphi \text { if and only if }(k, p) \models \mathcal{W} \varphi
$$

Proof It is easy to check that the construction satisfies the properties of a birelational model. To prove that the encoding preserves the forcing relation we proceed by induction on the formula $\varphi \in$ $\operatorname{Frm}(\mathrm{Pls})$. The statement of the proposition is easily verified on $\top, \perp$ and on atoms.

Induction hypothesis. We consider a formula $\varphi \in \operatorname{Frm}(\mathrm{Pls})$, and assume that the proposition holds for each of its sub-formulae. For sake of clarity, we just illustrate the cases of modalities @ $p$ and $\square$.

Case $\varphi=\varphi_{1} @ q$. Then $(k, p) \models \mathcal{K} \varphi$ means that $q \in P_{k}$ and $(k, q) \models \mathcal{K} \varphi_{1}$. By induction hypothesis and definition, this is equivalent to saying that there exists $(k, q) R^{\prime}(k, p)$ such that $(k, q) \downarrow q$, and $(k, q) \models \mathcal{W} \varphi_{1}$. This is equivalent to saying that $(k, p) \models \mathcal{W} \varphi_{1} @ q$.

Case $\varphi=\square \varphi_{1}$. Then $(k, p) \models_{\mathcal{K}} \varphi$ means that for every $l \geq k$ and every $q \in P_{l}$, we have $(l, q) \models \mathcal{K} \varphi_{1}$. By induction hypothesis and definition, this is equivalent to: for every $(l, p) \geq^{\prime}(k, p)$ and $(l, q) R^{\prime}(l, p)$, it is the case that $(l, q) \models_{\mathcal{W}} \varphi_{1}$. This is equivalent to $(k, p) \models_{\mathcal{W}} \square \varphi_{1}$. 
One thing worth pointing out is that in the resulting birelational model, the evaluation is total. It is easy to see the converse: every birelational model with a total evaluation can be encoded as a Kripke model such that the forcing relation is preserved. In the reverse encoding, the set of Kripke states is the set of equivalence classes under reachability, and the set of places associated to a class is the set of all the evaluations of its elements. Therefore, the class of Kripke models corresponds semantically to the class of birelational models in which the evaluation is total.

The encoding cannot be reversed if we consider birelational worlds with partial evaluation. Please note that this is not just a consequence of having undefined worlds in birelational models. If this was the case, we could have added "undefined" places in each Kripke state. The real issue is that when the evaluation is partial, two "undefined" worlds reachable from each other can be ordered: a situation that will be ruled out if the evaluation was total as a consequence of coherence and uniqueness. In Kripke models, "reachability" and order are essentially orthogonal. Hence, the reverse encoding will fail to preserve the forcing relation.

This is no accident, and as we have pointed out before, partiality of the evaluation in birelational models is essential for the proof of the finite model property. This was illustrated by the "finite model" $\mathcal{W}_{\text {exam }}$ in Example 9. In $\mathcal{W}_{\text {exam }}$, it is the case that $w_{1} \leq w_{2}, w_{1} R w_{2}, w_{1} \uparrow$ and $w_{2} \downarrow p$. As discussed there, this model allows us to refute the judgement ; $\square \neg \neg A \boldsymbol{a t} p \vdash\{p\} \neg \neg \square$ A at $p$. As we will see later, the judgement will be valid in every finite Kripke model.

The encoding and soundness of logic with respect to birelational models proves soundness of Kripke semantics, as detailed in the companion technical report [11].

Corollary 17 (Soundness) If $\Gamma ; \Delta \vdash^{P} \mu$ at $p$ is derivable in the logic, then it is valid in every Kripke model.

\section{Bounded contexts and Completeness}

In this section, we shall prove completeness of the logic with respect to both Kripke and birelational semantics. The proof will follow a modification of standard proofs of completeness of intuitionistic logics[20, 36, 7, 37], and we will construct a particular Kripke model: the canonical bounded Kripke model. The reason for the term "bounded" shall become clear later on. We will prove that a judgement is valid in the canonical bounded model if and only if it is derivable in the logic. Then we will use the encoding of the Kripke models into birelational models (see §3.2), and this will allow us to conclude completeness of birelational models. The resulting model will be used to prove the finite model property in $\S 5.3$. The construction of the model is adapted from [36].

We also point out that we shall prove the completeness results in the case where $P$ is finite. This is not a serious restriction for completeness, and the result can be extended to judgements where $P$ is infinite. The real advantage of using a finite set of places is that it will assist in the proof of finite model property as we will see in $\S 5$ (see Lemma 29).

We begin by defining sub-formulae of a pure formula. A sub-formula of a pure formula $\varphi$ is inductively generated as:

- $\varphi$ is a sub-formula of itself;

- if any of $\varphi_{1} \wedge \varphi_{2}, \varphi_{1} \vee \varphi_{2}$, and $\varphi_{1} \rightarrow \varphi_{2}$ is a sub-formula of $\varphi$, then so are $\varphi_{1}$ and $\varphi_{2}$; and

- if any of $\square \varphi_{1}, \nabla \varphi_{1}$, and $\varphi_{1} @ p$ is a sub-formula of $\varphi$, then so is $\varphi_{1}$. 
Given any set of pure formulae $\Theta$, the sub-formula closure $\Theta^{*}$, is the set of sub-formulae of each of its members. Formally:

$$
\Theta^{*} \stackrel{\text { def }}{=}\{\psi: \psi \text { is a subformula of } \varphi \in \Theta\} .
$$

We use sub-formulae closure to define Bounded contexts:

Definition 18 (Bounded Contexts) Given a finite set of places $P$ and a finite set of pure formulae $\Theta \in \operatorname{Frm}(P)$, a pair $(Q, \Delta)$ is a $(P, \Theta)$-bounded context if

- $Q$ is a finite set of places that contains $P$, i.e., $P \subseteq Q$; and

- $\Delta$ is a finite set of sentences of the form $\varphi$ at $q$ where $\varphi \in \Theta^{*}$ and $q \in Q$.

The bounded contexts will be used as Kripke states in the canonical model. However, we will need particular kinds of bounded contexts.

Definition 19 (Prime Bounded Contexts) Let $P$ be a finite set of places, and $\Theta, \Gamma \subseteq F r m(P)$ be two finite sets of pure formulae. A $(P, \Theta)$-bounded context $(Q, \Delta)$ is said to be $\Gamma$-prime if

- $\Gamma ; \Delta \vdash^{Q} \varphi$ at $q$ for $\varphi \in \Theta^{*}$ and $q \in Q$ implies that $\varphi$ at $q \in \Delta$ ( $\Theta$-deductive closure);

- $\Gamma ; \Delta \nvdash^{Q} \perp$ at $q$ for every $q \in Q$ (Consistency);

- $\Gamma ; \Delta \vdash^{Q} \varphi \vee \psi$ at $q$ for $\varphi \vee \psi \in \Theta^{*}$ and $q \in Q$ implies that either $\varphi$ at $q \in \Delta$ or $\psi$ at $q \in \Delta$ $(\Theta$-disjunction property); and

- $\Gamma ; \Delta \vdash^{Q} \diamond \varphi$ at $q$ for $\nabla \varphi \in \Theta^{*}$ and $q \in Q$ implies that there exists $q^{\prime} \in Q$ such that $\varphi$ at $q^{\prime} \in$ $\Delta(\Theta$-diamond property).

As an example, let $A$ be an atom. Let $P=\{p\}, \Theta=\{A @ p\}$ and $Q=\{p, q\}$. Consider the following sets of sentences:

- $\Delta_{1}=\{A$ at $p$, A at $q, A @ p$ at $p\}$;

- $\Delta_{2}=\{A \boldsymbol{a t} p, A$ at $q, A @ p$ at $p, A @ p$ at $q\} ;$ and

- $\Delta_{3}=\{A$ at $p, A$ at $q, A @ p$ at $p, A @ p$ at $q, \diamond A$ at $q\}$.

Clearly, we have that $P \subseteq Q$. If $\psi$ at $r$ is a sentence in $\Delta_{1}$ or $\Delta_{2}$, then $\psi$ is a sub-formula of $\Theta$ and $r \in Q$. Therefore, $\left(Q, \Delta_{1}\right)$ and $\left(Q, \Delta_{2}\right)$ are $(P, \Theta)$ - bounded contexts. On the other hand, $\left(Q, \Delta_{3}\right)$ is not a $(P, \Theta)$-bounded context as $\diamond A$ is not a sub-formula of $A @ p$.

If we let $\Gamma$ to be the list $\{A\}$, then it follows easily that $\Gamma ; \Delta_{1} \vdash^{Q}$ A at $p$. Using the inference rule of introduction of @, we get $\Gamma ; \Delta_{1} \vdash^{Q} A @ p$ at $q$. However, we have that $A @ p$ at $q \notin \Delta_{1}$. Therefore, $\left(Q, \Delta_{1}\right)$ is not $\Gamma$-prime. On the other hand, $\left(Q, \Delta_{2}\right)$ is $\Gamma$-prime.

The canonical model will be built by choosing the Kripke states to be prime bounded contexts. We will first show that bounded contexts can be extended to prime bounded contexts. Before we proceed, we state a proposition that says that the cut-rule is admissible in the logic. In [17], this has been proved for the logic without the disjunctive connectives. The proof can be extended for the logic with disjunctive connectives:

Proposition 20 If $\Gamma ; \Delta \vdash^{P} \mu$ at $p_{1}$ and $\Gamma ; \Delta$, $\mu$ at $p_{1} \vdash^{P} \psi$ at $p$ then $\Gamma ; \Delta \vdash^{P} \psi$ at $p$. 
Lemma 21 (Prime Bounded Extension) Let $(Q, \Delta)$ be a $(P, \Theta)$-bounded context and $\psi$ be a pure formula in $\operatorname{Frm}(P)$. Given a finite subset $\Gamma \subseteq \operatorname{Frm}(P)$ and $q \in Q$ such that $\Gamma ; \Delta \nvdash^{Q} \psi$ at $q$, there exists a $(P, \Theta)$-bounded context $\left(Q^{\prime}, \Delta^{\prime}\right)$ such that

1. $\left(Q^{\prime}, \Delta^{\prime}\right)$ is $\Gamma$-prime,

2. $\left(Q^{\prime}, \Delta^{\prime}\right)$ extends $(Q, \Delta)$, i.e., $Q \subseteq Q^{\prime}$, and $\Delta \subseteq \Delta^{\prime}$, and

3. $\Gamma ; \Delta^{\prime} \nvdash^{Q^{\prime}} \psi$ at $q$.

Proof Please note that by definition $P, \Theta$ and $\Theta^{*}$ are finite sets. Pick new places $q_{\diamond \varphi}$, one for each formula $\diamond \varphi \in \Theta^{*}$. Let $Q_{\diamond}$ be the set of all such places. As the set $\Theta^{*}$ is finite, $Q_{\diamond}$ is also a finite set. Finally, let $\Sigma$ be the set of sentences $\varphi$ at $q$ such that $\varphi \in \Theta^{*}$ and $q \in Q \cup Q_{\diamond}$. As $\Theta^{*}, Q$ and $Q_{\diamond}$ are finite sets, $\Sigma$ is also finite.

The set $\Delta^{\prime}$ required in the lemma would be a subset of $\Sigma$ and the set $Q^{\prime}$ would be a subset of $Q \cup Q_{\diamond}$. These sets would be obtained by a series of extensions $\Delta_{n}, Q_{n}$ which will satisfy certain properties:

Property 1 For every $n \geq 0$

1. $Q_{n} \subseteq Q \cup Q_{\diamond}$ and $\Delta_{n} \subseteq \Sigma$;

2. $Q_{n} \subseteq Q_{n+1}, \Delta_{n} \subseteq \Delta_{n+1}$;

3. $\left(Q_{n}, \Delta_{n}\right)$ is $(P, \Theta)$-bounded context; and

4. $\Gamma ; \Sigma_{n} \nvdash^{Q_{n}} \psi$ at $q$.

The series is constructed inductively. In the induction at an odd step, we will create a witness for a formula of the type $\diamond \varphi$. At an even step, we deal with disjunction property. We shall also construct two sets:

- treated $_{n}^{\diamond}$, that will be the set of the formulae $\diamond \varphi \in \Theta^{*}$ for which we have already created a witness.

- treated $_{n}^{\vee}$, that will be the set of the formulae $\psi_{1} \vee \psi_{2}$ at $q \in \Sigma$ which satisfy the disjunction property.

We pick an enumeration of $\Theta^{*}$, and fix it. We start off by defining treated $d_{0}^{\diamond}=\emptyset$, treated $_{0}^{\vee}=\emptyset$, $Q_{0}=Q$, and $\Delta_{0}=\Delta$. It is clear from the hypothesis of the lemma that $Q_{0}$ and $P_{0}$ satisfy the four points of Property1.

Then we proceed inductively, and assume that $Q_{n}, \Delta_{n}(n \geq 0)$ have been constructed satisfying Property 1. In step $n+1$, we consider two cases:

1. If $n+1$ is odd, then pick the first formula $\psi_{1} \vee \psi_{2} \in \Theta^{*}$ in the enumeration of $\Theta^{*}$, such that

- $\Gamma ; \Delta_{n} \vdash^{Q_{n}} \psi_{1} \vee \psi_{2}$ at $r$, for some $r \in Q_{n}$;

- $\psi_{1} \vee \psi_{2}$ at $r \notin$ treated ${ }_{n}^{\vee}$.

If no such formula exists, then let $Q_{n+1}=Q_{n}$ and $\Delta_{n+1}=\Delta_{n}$. In this case $Q_{n+1}$ and $\Delta_{n+1}$ satisfy the four points of Property 1 by induction. 
Otherwise, if both $\Gamma ; \Delta_{n}, \psi_{1}$ at $r \vdash^{Q_{n}} \psi \boldsymbol{a t} q$ and $\Gamma ; \Delta_{n}, \psi_{2} \boldsymbol{a t} r \vdash^{Q_{n}} \psi \boldsymbol{a t} q$ then we can deduce $\Gamma ; \Delta_{n} \vdash^{Q_{n}} \psi \boldsymbol{a t} q$. However, we have that $\Delta_{n}, Q_{n}$ satisfy Property 1 . Hence, it must be the case that either $\Gamma ; \Delta_{n}, \psi_{1}$ at $r \nvdash^{Q_{n}} \psi$ at $q$ or $\Gamma ; \Delta_{n}, \psi_{2}$ at $r \nvdash^{Q_{n}} \psi$ at $q$.

We define $\Delta_{n+1}=\Delta_{n} \cup\left\{\psi_{1}\right.$ at $\left.r\right\}$ if $\Gamma ; \Delta_{n}, \psi_{1}$ at $r \nvdash^{Q_{n}} \psi$ at $p$, and $\Delta_{n+1}=\Delta_{n} \cup\left\{\psi_{2}\right.$ at $\left.r\right\}$ otherwise. We define $Q_{n+1}=Q_{n}$. We have by construction $Q_{n} \subseteq Q_{n+1}, Q_{n+1} \subseteq Q \cup Q_{\diamond}$ and $\Delta_{n} \subseteq \Delta_{n+1}$.

We have $r \in Q_{n}$. By definition, the set $\Theta^{*}$ is closed under sub-formulae. Therefore as $\psi_{1} \vee \psi_{2} \in \Theta^{*}$, we have both $\psi_{1}$ and $\psi_{2}$ are in $\Theta^{*}$. This implies that $\psi_{1} \boldsymbol{a t} r$ and $\psi_{1} \boldsymbol{a t} r$ are in $\Sigma$, and $\left(Q_{n+1}, \Delta_{n}\right)$ is $(P, \Theta)$-bounded context.

Also by construction $\Gamma ; \Delta_{n+1} \nvdash_{n+1}^{Q} \psi$ at $q$. Therefore, $Q_{n+1}, \Delta_{n+1}$ satisfies Property 1. Finally, we let treated $_{n+1}^{\vee}=$ treated $_{n}^{\vee} \cup\left\{\psi_{1} \vee \psi_{2}\right.$ at $\left.r\right\}$ and treated $_{n+1}^{\diamond}=$ treated $_{n}^{\diamond}$.

2. If $n+1$ is even, pick the first formula $\nabla \varphi$ in the enumeration of $\Theta^{*}$ such that

- $\Gamma ; \Delta_{n} \vdash^{Q_{n}} \diamond \varphi$ at $r$, for some $r \in Q_{n}$;

- $\diamond \varphi \notin$ treated $_{n}^{\diamond}$.

Let $Q_{n+1}=Q_{n}+q_{\diamond \varphi}, \Delta_{n+1}=\Delta_{n} \cup\left\{\varphi\right.$ at $\left.q_{\diamond \varphi}\right\}$, treated $_{n+1}=$ treated $_{n} \cup\{\diamond \varphi\}$ and treated $\mathrm{v}_{n+1}^{\vee}=$ treated $_{n}^{\vee}$. We have by construction that $Q_{n+1}$ and $\Delta_{n+1}$ satisfy the first three points of Property1. We claim that $\Gamma ; \Delta_{n+1} \nvdash^{Q_{n+1}} \psi \boldsymbol{a t} q$ also.

Suppose that $\Gamma ; \Delta_{n+1} \vdash^{Q_{n+1}} \psi$ at q, i.e., $\Gamma ; \Delta_{n}, \varphi$ at $q_{\diamond \varphi} \vdash^{Q+q_{\diamond \varphi}} \psi$ at $q$. We also have that $\Gamma ; \Delta_{n} \vdash^{Q_{n}} \diamond \varphi$ at $r$. In fact, by the inference rule $\diamond E$ :

$$
\frac{\Gamma ; \Delta_{n} \vdash^{Q_{n}} \diamond \varphi \text { at } r \quad \Gamma ; \Delta_{n}, \varphi \text { at } q_{\diamond \varphi} \vdash^{Q+q \diamond \varphi} \psi \text { at } q}{\Gamma ; \Delta_{n} \vdash^{Q_{n}} \psi \text { at } q} \diamond E
$$

This contradicts the hypothesis on $Q_{n}, \Delta_{n}$. Hence $\Gamma ; \Delta_{n+1} \nvdash^{Q_{n+1}} \psi$ at $q$. Therefore, $Q_{n+1}$ and $\Delta_{n+1}$ satisfy Property1.

Therefore, we get by construction that $Q_{n}, \Delta_{n}$ satisfy Property 1 . We define $Q^{\prime}=\bigcup_{n \geq 0} Q_{n}$, and $\Delta^{\prime \prime}=\bigcup_{n>0} \Delta_{n}$. Now, using Property $1, Q^{\prime} \subseteq Q \cup Q_{\diamond}$ and $\Delta^{\prime \prime} \subseteq \Sigma$. This implies that $Q^{\prime}$ and $\Delta^{\prime \prime}$ are finite sets. (Note that this means that the series $\left(Q_{n}, \Delta_{n}\right)$ is eventually constant). Using Property 1 , we can easily show that $\left(Q^{\prime}, \Delta^{\prime \prime}\right)$ is a $(P, \Theta)$ - bounded context and $\Gamma ; \Delta^{\prime \prime} \nvdash^{Q^{\prime}} \psi$ at $q$.

Finally, we define $\Delta^{\prime}$ to be the set of all sentences $\varphi$ at $s \in \Sigma$ such that $\Gamma ; \Delta^{\prime \prime} \vdash^{Q^{\prime}} \varphi$ at $s$. As a consequence of cut rule, we get that

$$
\Gamma ; \Delta^{\prime} \vdash Q^{Q^{\prime}} \mu \boldsymbol{a t} r \text { if and only if } \Gamma ; \Delta^{\prime \prime} \vdash^{Q^{\prime}} \mu \boldsymbol{a t} r
$$

Clearly, $\Delta^{\prime}$ extends $\Delta^{\prime \prime}$ and hence $\Delta$. Furthermore, by construction $\left(Q^{\prime}, \Delta^{\prime}\right)$ is $(P, \Theta)$-bounded. Also we get $\Gamma ; \Delta^{\prime} \nvdash^{Q^{\prime}} \psi$ at $q$, thanks to the equivalence (5). Finally, $\left(Q^{\prime}, \Delta^{\prime}\right)$ can be easily shown to be $\Gamma$-prime by construction.

We finally construct the bounded canonical model. In the model, the set of Kripke states is the set of prime bounded contexts $(Q, \Delta)$ ordered by inclusion. A place belongs to the state $(Q, \Delta)$ only if it is in $Q$, and an atom $A$ is placed in a place $r$ in the state $(Q, \Delta)$ only if $A$ at $r \in \Delta$. More formally, we have: 
Definition 22 (Bounded Canonical Model) Given a finite set of places $P$ and two finite sets of pure formulae $\Theta, \Gamma \subseteq \operatorname{Frm}(P)$, the $\Gamma$-prime and $(P, \Theta)$-bounded canonical model is the quadruple $\mathcal{K}_{\text {can }} \stackrel{\text { def }}{=}\left(K, \leq,\left\{P_{k}\right\}_{k \in K},\left\{I_{k}\right\}_{k \in K}\right)$, where

- the set $K$ is the set of all $(P, \Theta)$-bounded contexts that are $\Gamma$-prime;

- $\left(Q_{1}, \Delta_{1}\right) \leq\left(Q_{2}, \Delta_{2}\right)$ if and only if $Q_{1} \subseteq Q_{2}$ and $\Delta_{1} \subseteq \Delta_{2}$; and

- $P_{(Q, \Delta)} \stackrel{\text { def }}{=} Q$;

- for $k=(Q, \Delta)$, the function $I_{k}:$ Atoms $\rightarrow \operatorname{Pow}\left(P_{k}\right)$ is defined as

$$
I_{(Q, \Delta)}(A) \stackrel{\text { def }}{=}\{q \in Q: \text { A at } q \in \Delta\} .
$$

Given a finite set of places $P$ and a finite set of formulae $\Gamma \in \operatorname{Frm}(P)$, we say that $\Gamma$ is consistent if $\Gamma ; \nvdash^{P} \perp$ at $p$ for any $p \in P$. If $\Gamma$ is consistent, then Lemma 21 guarantees that the set of states in the canonical model is non-empty. This ensures that the bounded canonical model is a Kripke model:

Lemma 23 (Canonical Evaluation) Given a finite set places $P$ and two finite sets of pure formulae $\Theta, \Gamma \in \operatorname{Frm}(P)$ such that $\Gamma$ is consistent, let $\mathcal{K}_{c a n}$ be the $\Gamma$-prime and $(P, \Theta)$-bounded canonical model. Then

1. $\mathcal{K}_{c a n}$ is a Kripke model; and

2. if $\models_{\mathcal{K}}$ is the forcing relation on $\mathcal{K}_{c a n}$, then for every $\varphi \in \Theta^{*},(Q, \Delta) \in K$ and $q \in Q$ :

$$
(Q, \Delta) \models_{\mathcal{K}} \varphi \boldsymbol{a t} q \text { if and only if } \varphi \text { at } q \in \Delta .
$$

Proof Clearly, all the properties required for a Kripke model are verified. The proof for part 2 is a standard induction on the structure of formulae.

We are now ready to prove completeness. It will imply the completeness theorem for birelational models as a corollary.

Theorem 24 (Completeness) If $P$ is finite and the judgement $\Gamma ; \Delta \vdash^{P} \varphi$ at $p$ is valid in every Kripke model, then it is provable in the logic.

Proof Assume that $\Gamma ; \Delta \models^{P} \varphi$ at $p$ is valid. We have:

1. $\operatorname{PL}(\Gamma) \cup \operatorname{PL}(\Delta) \cup \operatorname{PL}(\varphi) \cup\{p\} \subseteq P$.

2. If $\mathcal{K}=\left(K, \leq,\left\{P_{k}\right\}_{k \in K},\left\{I_{k}\right\}_{k \in K}\right)$ is a Kripke model, then for every $k \in K$ such that $P \subseteq P_{k}$,

$$
k \models \varphi \text { at } p \text { whenever } k \models \Gamma ; \Delta .
$$

We need to show that $\Gamma ; \Delta \vdash^{P} \varphi$ at $p$.

Assume that $\Gamma ; \Delta \nvdash^{P} \varphi$ at $p$. We fix $\Theta \stackrel{\text { def }}{=}\{\square \psi: \psi \in \Gamma\} \cup\{\mu: \mu$ at $q \in \Delta\} \cup\{\varphi\}$. Please note that $\Theta \in \operatorname{Frm}(P)$ and $(P, \Delta)$ is a $(P, \Theta)$-bounded context. By Lemma 21, there is a $\Gamma$-prime and 
$(P, \Theta)$ - bounded context $(Q, \Sigma)$ extending $(P, \Delta)$ such that $\Gamma ; \Sigma \nvdash^{Q} \varphi$ at $p$. We get $\varphi$ at $p \notin \Sigma$. Fix $(Q, \Sigma)$.

Now consider the $\Gamma$-prime and $(P, \Theta)$-bounded canonical model $\mathcal{K}_{c a n}$ as constructed in Definition 22, and let $=_{\mathcal{K}}$ be the forcing relation in $\mathcal{K}_{\text {can }}$. Consider the Kripke state $(Q, \Sigma)$. We claim that $(Q, \Sigma) \models_{\mathcal{K}} \Gamma ; \Delta$.

Pick $\psi \in \Gamma, r \in Q$ and fix them. We first show that $\Gamma ; \Sigma \vdash^{Q} \square \psi$ at $r$. In the proof, we first choose a new place $m \notin Q$, and then use the inference rule $G$ to conclude that $\psi$ at $r$ is derivable from $\Gamma, \Sigma$. We then use the inference rule $\square I$ to obtain $\Gamma ; \Sigma \vdash^{Q} \square \psi$ at $r$. More formally,

$$
\frac{\overline{\Gamma ; \Sigma \vdash^{Q+m} \psi \boldsymbol{a t} m}}{\Gamma ; \Sigma \vdash^{Q} \square \psi \boldsymbol{a t} r} \square I
$$

As $\psi \in \Gamma$, we have that $\square \psi \in \Theta$. As $r \in Q$, we have by definition of prime contexts, $\square \psi$ at $r \in \Sigma$. Using Lemma 23, we get that $(Q, \Sigma) \models_{\mathcal{K}} \square \psi$ at $r$.

Furthermore, $\Delta$ is contained in $\Sigma$. Therefore, by Lemma $23,(Q, \Sigma) \models_{\mathcal{K}} \mu$ at $q$ whenever $\mu$ at $q \in \Delta$.

Hence, we get that the Kripke state $(Q, \Sigma) \models \Gamma ; \Delta$. By our assumption, we get $(Q, \Sigma) \models \mathcal{K}$ $\varphi$ at $p$ also. By Lemma 23, we get $\varphi$ at $p \in \Sigma$. However our choice of $Q, \Sigma$ was such that $\varphi$ at $p \notin$ $\Sigma$. We have just reached a contradiction, and hence we can conclude that $\Gamma ; \Delta \vdash^{P} \varphi$ at $p$.

Now, by the encoding of Kripke models into birelational models (see Proposition 16), if a judgement is valid in all birelational models then it is valid in all Kripke models. As the class of Kripke models is complete, we get that the class of birelational models is also complete for the logic.

Corollary 25 If $P$ is finite and the judgement $\Gamma ; \Delta \vdash^{P} \varphi$ at $p$ is bi-valid in every birelational model, then it is provable in the logic.

The proofs in this section can be suitably modified to allow $P$ to be infinite, as they do not actually require context sets to be finite. Finiteness is actually required for the proof of the finite model property, and not for completeness.

There is another way in which we can deduce the completeness results when $P$ is infinite. For this, we take recourse to the following proposition which states that to derive a judgment, it is sufficient just to consider the set of places appearing in the formulae of the judgement itself. This was proved for the logic without disjunctive connectives in [17], and the proof can be extended for the whole logic.

Proposition 26 Let $P_{0}=\operatorname{PL}(\Gamma) \cup \operatorname{PL}(\Delta) \cup \operatorname{PL}(\varphi) \cup\{p\}$, and $P_{0} \subseteq P$. Then $\Gamma ; \Delta \vdash^{P} \varphi$ at $p$ if and only if $\Gamma ; \Delta \vdash^{P_{0}} \varphi$ at $p$.

In order to use completeness result for judgements in which $P$ is infinite, we proceed as follows. Suppose that

$$
\Gamma ; \Delta \nvdash^{P} \varphi \text { at } p .
$$

Let $P_{0}=\operatorname{PL}(\Gamma) \cup \operatorname{PL}(\Delta) \cup \operatorname{PL}(\varphi) \cup\{p\}$. Please observe that by the above Proposition, we get

$$
\Gamma ; \Delta \nvdash^{P_{0}} \varphi \text { at } p \text {. }
$$

Using Theorem 24, we get a Kripke world $\mathcal{K}$ with a Kripke state $k$ such that $k$ forces $\Gamma ; \Delta$ but not $\varphi$ at $p$. Furthermore, $k$ has at least $P_{0}$ places. Without loss of generality, we can assume that $\mathcal{K}$ does not contain any place in the set $P \backslash P_{0}$ (otherwise we can rename them). Now pick $p_{0} \in P$, and fix it. In each Kripke state of $\mathcal{K}$ add new places $P \backslash P_{0}$, each duplicating $p_{0}$. It can be shown that in the resulting model the Kripke state $k$ still forces $\Gamma ; \Delta$ but not $\varphi$ at $p$. Therefore, we obtain completeness for Kripke semantics when $P$ is infinite. 


\section{Finite Model Property}

In this section, we will show that if a judgement is not provable in the logic, then there is a finite birelational model that invalidates it. The proof will use the counter-model from the proof of completeness in $\S 4$. The birelational model constructed in the proof of completeness consists of worlds of the form $(Q, \Delta, q)$ where $(Q, \Delta)$ are prime bounded contexts and $q \in Q$. The model constructed may be infinite as it may contain infinitely many worlds. However, by using techniques similar to those used in [36], we will be able to construct a finite model that is equivalent to the counter-model. The key technique in the construction is the identification of triples $(Q, \Delta, q)$ that differ only in renaming of places other than those in $P$. We start the proof by discussing renaming functions.

\subsection{Renaming functions}

Given any two sets of places $Q_{1}, Q_{2}$, a renaming function is a function $f: Q_{1} \rightarrow Q_{2}$. Intuitively, $f$ renames a place $q$ in $Q_{1}$ as $f(q)$. Given a renaming function $f: Q_{1} \rightarrow Q_{2}$, we can extend $f$ to a function from the set $\operatorname{Frm}\left(Q_{1}\right)$ into the set $\operatorname{Frm}\left(Q_{2}\right)$ by replacing all occurrences of places $q$ by $f(q)$. More formally,

- $f(A) \stackrel{\text { def }}{=} A$ for all atoms $A$;

- $f\left(\varphi_{1} \circ \varphi_{2}\right) \stackrel{\text { def }}{=} f\left(\varphi_{1}\right) \circ f\left(\varphi_{2}\right)$ for $\circ \in\{\vee, \wedge, \rightarrow\}$;

- $f(\varphi @ q) \stackrel{\text { def }}{=} f(\varphi) @ f(q)$;

- $f(\diamond \varphi) \stackrel{\text { def }}{=} \diamond f(\varphi)$ and $f(\square \varphi) \stackrel{\text { def }}{=} \square f(\varphi)$.

This can be further extended to contexts $\Gamma ; \Delta$ by applying $f$ to all formulae in $\Gamma$ and all sentences in $\Delta$ with $f$ extended to sentences as $f(\varphi$ at $q) \stackrel{\text { def }}{=} f(\varphi)$ at $f(q)$.

If $f$ is a renaming function, then we can transform a proof of a judgement $\Gamma ; \Delta \vdash^{Q_{1}} \varphi$ at $q$ to a proof of the judgement $f(\Gamma ; \Delta) \vdash^{Q_{2}} f(\varphi)$ at $f(q)$ :

Lemma 27 (Provability Preservation Under Renaming) Let $f: Q_{1} \rightarrow Q_{2}$ be a renaming function. Then for any set of pure formulae $\Gamma$, any set of sentences $\Delta$, any formula $\varphi$ and any place $q$ such that $\operatorname{PL}(\Gamma) \cup \operatorname{PL}(\Delta) \cup \operatorname{PL}(\varphi) \cup\{q\} \subseteq Q_{1}$, we have:

$$
\Gamma ; \Delta \vdash^{Q_{1}} \varphi \text { at } q \text { implies } f(\Gamma ; \Delta) \vdash^{Q_{2}} f(\varphi) \text { at } f(q) \text {. }
$$

Proof Intuitively, replace all occurrences of places $r$ in the proof of $\Gamma ; \Delta \vdash^{Q_{1}} \varphi$ at $q$ by $f(r)$ to obtain a proof of $f(\Gamma ; \Delta) \vdash^{Q_{2}} f(\varphi)$ at $f(q)$. This can be shown inductively on the length of the proof of $\Gamma ; \Delta \vdash^{Q_{1}} \varphi$ at $q$.

For example, let $Q_{1}=\{p, q\}$ and $Q_{2}=\{r\}$. Let $f: Q_{1} \rightarrow Q_{2}$ be the function $f(p)=$ $r, f(q)=r$. Let $A$ be an atom, and let $\Gamma$ to be the empty list. We have $\Gamma$; $A$ at $p \vdash^{Q_{1}} A @ p$ at $q$. Then by the Lemma 27, $\Gamma ; A$ at $r \vdash^{Q_{2}} A @ r$ at $r$.

\subsection{Pointed Contexts and Morphisms}

Let $P, Q$ be finite sets of places such that $P \subseteq Q$. Let $\Theta \subseteq \operatorname{Frm}(P)$ be a finite set of pure formulae with sub-formula closure $\Theta^{*}$. Please recall that given a finite set of sentences $\Delta$, we say that $(Q, \Delta)$ 
is a $(P, \Theta)$-bounded context if for every sentence $\varphi$ at $r$ it is the case that $\varphi \in \Theta^{*}$ and $r \in Q$. Given a $(P, \Theta)$-bounded context $(Q, \Delta)$, we will say that $(Q, \Delta, q)$ is a pointed $(P, \Theta)$-bounded context if $q \in Q$. Henceforth, we refer to such triples as $(P, \Theta)$-pcontexts. The element $q$ is said to be the point of the pcontext $(Q, \Delta, q)$. Following [36], we lift the notion of renaming functions to morphisms between pcontexts:

Definition 28 (Morphism) Let $w_{1}$ and $w_{2}$ be two $(P, \Theta)$-pcontexts, and let $w_{i}=\left(Q_{i}, \Delta_{i}, q_{i}\right)$ for $i=1,2$. A morphism from $w_{1}$ to $w_{2}$ is a renaming function $f: Q_{1} \rightarrow Q_{2}$ such that

1. $f(p)=p$ for every $p \in P$;

2. if $\varphi$ at $q \in \Delta_{1}$ then $\varphi$ at $f(q) \in \Delta_{2}$; and

3. $f\left(q_{1}\right)=q_{2}$.

We write $w_{1} \precsim w_{2}$ whenever there is a morphism from $w_{1}$ to $w_{2}$. Furthermore, we write $w_{1} \curvearrowleft w_{2}$ if $w_{1} \precsim w_{2}$ and $w_{2} \precsim w_{1}$.

The first part of the definition says that the renaming function does not change the places in $P$. Now for every sentence $\varphi$ at $q \in \Delta_{1}$, it is the case that $\varphi \in \operatorname{Frm}(P)$. Therefore, the second condition is equivalent to saying that $f\left(\Delta_{1}\right) \subseteq \Delta_{2}$. Hence, $\left(Q_{1}, \Delta_{1}, q_{1}\right) \precsim\left(Q_{2}, \Delta_{2}, q_{2}\right)$ intuitively means that $\Delta_{2}$ has "more" sentences than $\Delta_{1}$ up-to renaming. Finally, the third part says that a morphism preserves the point of a pcontext.

For example, let $P=\{p\}, \Theta=\{A\}$ and $Q_{1}=Q_{2}=\{p, q, r\}$. Let $f: Q_{1} \rightarrow Q_{2}$ be the renaming function defined as $f(p)=p, f(q)=r$ and $f(r)=q$. Consider the three sets of sentences:

- $\Delta_{1}=\Delta_{2}=\{$ A at $q$, A at $p\}$, and

- $\Delta^{\prime}=\{$ A at $p, A$ at $r\}$.

We have $f(A$ at $q)=A$ at $r$. Now, we have that $A$ at $r \notin \Delta_{2}$ and $A$ at $r \in \Delta^{\prime}$. Therefore, $f$ is not a morphism from $\left(Q_{1}, \Delta_{1}\right)$ to $\left(Q_{2}, \Delta_{2}\right)$. On the other hand, $f$ is a morphism from $\left(Q_{1}, \Delta_{1}\right)$ to $\left(Q_{2}, \Delta^{\prime}\right)$.

Clearly, $\precsim$ is a preorder. The identity function gives reflexivity and function composition gives transitivity. This makes the relation $\simeq$ an equivalence relation. If $w$ is a pcontext, then we shall use $[w]$ to denote the class of the pcontexts equivalent to $w$ with respect to the relation $\backsim$. We shall use these equivalence classes as the worlds of the finite counter-model. The order amongst the worlds will be given by the preorder $\precsim$. We will now show that the relation $\simeq$ partitions the set of pcontexts into finite number of classes. Please note that it is in this proof, we use the fact that the set $P$ is finite:

Lemma 29 (Finite Partition) The set of $(P, \Theta)$-pcontexts is partitioned into a finite number of equivalence classes by the equivalence $\simeq$.

Proof We will show that every $(P, \Theta)$-pcontext is equivalent to a canonical pcontext. The set of canonical pcontexts will be finite. Before we proceed, please note that $P$ and $\Theta$ are finite sets by definition. Hence, the sub-formula closure $\Theta^{*}$ and the powerset $\operatorname{Pow}\left(\Theta^{*}\right)$ must be finite sets.

We will now define the set of canonical pcontexts. For each $\Lambda \subseteq \Theta^{*}$ we choose a new place $\boldsymbol{r}_{\Lambda} \notin P$ such that $\boldsymbol{r}_{\Lambda_{1}} \neq \boldsymbol{r}_{\Lambda_{2}}$ if $\Lambda_{1} \neq \Lambda_{2}$. Let $R \stackrel{\text { def }}{=}\left\{\boldsymbol{r}_{\Lambda}: \Lambda \subseteq \Theta^{*}\right\}$. The cardinality of $R$ is the 
same as the cardinality of $\operatorname{Pow}\left(\Theta^{*}\right)$, and hence $R$ is finite. A canonical pcontext will have places amongst $P \cup R$. Furthermore, the canonical pcontext will contain the sentence $\varphi \boldsymbol{a t} \boldsymbol{r}_{\Lambda}$ if and only if $\boldsymbol{r}_{\Lambda}$ is a place in the pcontext and $\varphi \in \Lambda$. More formally, we say that the triple $(Q, \Sigma, q)$ is a canonical $(P, \Theta)$-pcontext if

- $Q$ is a set of places such that $P \subseteq Q \subseteq P \cup R$.

- $\Delta$ is the union of two sets $\Delta_{P}$ and $\Delta_{R}$, where

1. $\Delta_{P}$ is a set of sentences such that $\varphi$ at $s \in \Delta_{P}$ means that $\varphi \in \Theta^{*}$ and $s \in P$; and

2. $\Delta_{R}$ is the set of all sentences $\varphi$ at $\boldsymbol{r}_{\Lambda}$, where $\varphi \in \Lambda$ and $\boldsymbol{r}_{\Lambda} \in Q \cap R$. In other words, $\Delta_{R} \stackrel{\text { def }}{=}\left\{\varphi\right.$ at $\left.\boldsymbol{r}_{\Lambda}: \varphi \in \Lambda, \boldsymbol{r}_{\Lambda} \in Q \cap R\right\}$.

- $q \in Q$.

Clearly, a triple that satisfies the above points is a $(P, \Theta)$-pcontext. Furthermore, as the sets $P, R, \Theta^{*}$ are finite, the set of canonical pcontexts must be finite also.

We will now show that for every pcontext $w=(Q, \Delta, q)$ there is a canonical pcontext equivalent to it. This would immediately give us that the number of equivalence classes induced by $\simeq$ is finite.

Let $w=(Q, \Delta, q)$ be a $(P, \Theta)$-pcontext. Fix $w$. For $s \in Q$, let $H(s) \subseteq \Theta^{*}$ be the set of formulae $\varphi$ such that $\varphi$ at $s \in \Delta$.

We now define $w^{\prime}=\left(Q^{\prime}, \Delta^{\prime}, q^{\prime}\right)$, the canonical pcontext equivalent to $w$ as follows. $P$ will be contained in $Q^{\prime}$. For each $s \in Q \backslash P$, we add the place $\boldsymbol{r}_{H(s)}$ to $Q^{\prime}$. For $p \in P$, a sentence $\varphi$ at $p$ will be in $\Delta^{\prime}$ only if it is in $\Delta$. A sentence $\varphi \boldsymbol{a t} \boldsymbol{r}_{H(s)}$ will be in $Q^{\prime}$ only if $\varphi \in H(s)$. Finally, the point $q^{\prime}$ will be $q$ if $q \in P$. Otherwise the point $q^{\prime}$ will be $\boldsymbol{r}_{H(q)}$. More formally, we define:

- $Q^{\prime} \stackrel{\text { def }}{=} P \cup\left\{\boldsymbol{r}_{H(s)}: s \in Q \backslash P\right\}$

- $\Delta^{\prime} \stackrel{\text { def }}{=} \Delta_{P} \cup \Delta_{R}$, where

$$
\begin{aligned}
& -\Delta_{P} \stackrel{\text { def }}{=}\{\varphi \text { at } p: \varphi \text { at } p \in \Delta \text { and } p \in P\} \\
& -\Delta_{R} \stackrel{\text { def }}{=}\left\{\varphi \text { at } \boldsymbol{r}_{H(s)}: s \in Q \backslash P \text { and } \varphi \in H(s)\right\} \\
\text { - } q^{\prime} & \stackrel{\text { def }}{=} \begin{cases}q & \text { if } q \in P ; \\
\boldsymbol{r}_{H(q)} & \text { if } q \in Q \backslash P .\end{cases}
\end{aligned}
$$

Clearly, $\left(Q^{\prime}, \Delta^{\prime}, q^{\prime}\right)$ is a canonical $(P, \Theta)$-pcontext. Moreover, the renaming functions

$$
\begin{array}{ll}
f: Q \longrightarrow Q^{\prime} & f(s) \stackrel{\text { def }}{=} \begin{cases}s & \text { if } s \in P ; \\
\boldsymbol{r}_{H(s)} & \text { otherwise. }\end{cases} \\
g: Q^{\prime} \longrightarrow Q & g(t) \stackrel{\text { def }}{=} \begin{cases}t & \text { if } t \in P ; \\
q & \text { if } t=q^{\prime} ; \\
l & \text { otherwise, where } l \in Q \backslash P \text { is chosen s.t. } \\
& t=\boldsymbol{r}_{H(l)} .\end{cases}
\end{array}
$$

are morphisms from $w$ to $w^{\prime}$ and from $w^{\prime}$ to $w$, respectively. We conclude that $w \simeq w^{\prime}$. 


\subsection{The Finite Counter-Model}

Given a finite set of places $P$ and two finite sets of pure formulae $\Gamma, \Theta \subseteq \operatorname{Frm}(P)$, let $\mathcal{K}_{\text {can }}$ be the $\Gamma$-prime and $(P, \Theta)$-bounded canonical Kripke model as defined in $\S 4$ (see Definition 22). Let $\mathcal{W}_{c a n}=(W, \leq, R, I, E v a l)$ be the $\mathcal{K}_{c a n}$-birelational model obtained by using the encoding of $\mathcal{K}_{\text {can }}$ into a birelational model (see $\left.\S 3.2\right)$. We call $\mathcal{W}_{\text {can }}$ the $\Gamma$-prime and $(P, \Theta)$-bounded canonical birelational model. Please recall from the proof of completeness (see $\S 4$ ) that if a judgement $\Gamma ; \Sigma \vdash^{P} \varphi$ at $p$ is not provable, then $\mathcal{W}_{\text {can }}$ provides the birelational counter-model for the judgement for an appropriate choice of $\Theta$.

The worlds of $\mathcal{W}_{\text {can }}$ are pcontexts $(Q, \Delta, q)$ where $(Q, \Delta)$ are $\Gamma$-prime and $(P, \Theta)$-bounded. Two worlds $w_{1}=\left(Q_{1}, \Delta_{1}, q_{1}\right)$ and $w_{2}=\left(Q_{2}, \Delta_{2}, q_{2}\right)$ are reachable from each other if $Q_{1}=Q_{2}$ and $\Delta_{1}=\Delta_{2}$. Furthermore, $\left(Q_{1}, \Delta_{1}, q_{1}\right) \leq\left(Q_{2}, \Delta_{2}, q_{2}\right)$ if $Q_{1} \subseteq Q_{2}, \Delta_{1} \subseteq \Delta_{2}$ and $q_{1}=q_{2}$. A world $w=(Q, \Delta, q) \in I(A)$ for some atom $A$ if $A$ at $q \in \Delta$. The evaluation is a total function, and $E((Q, \Delta, q))=q$. Furthermore, as a consequence of definition of canonical models, a world $w=(Q, \Delta, q)$ forces a formula $\varphi \in \Theta^{*}$ if and only if $\varphi$ at $q \in \Delta$.

Even though the worlds in canonical birelational are composed of bounded pcontexts, the set of the worlds may itself be infinite. Following [36], we shall construct a finite model, called the quotient model, equivalent to the canonical model. For this model, we will use morphisms between pcontexts. Please recall that given pcontexts $w_{1}$ and $w_{2}, w_{1} \precsim w_{2}$ if there is a morphism from $w_{1}$ into $w_{2}$, and $w_{1} \curvearrowleft w_{2}$ if $w_{1} \precsim w_{2}$ and $w_{2} \precsim w_{1}$. The relation $\precsim$ is a preorder and $\backsim$ is an equivalence. The set of equivalence classes generated by $\simeq$ is finite by Lemma 29 . We write $[w]$ for the equivalence class of $w$.

In the quotient canonical model, the set of worlds will be $W / \simeq$, the set of equivalence classes generated by $\simeq$ on $W$. We have that $W / \bumpeq$ is finite. Our construction will ensure that $w$ in the canonical birelational model forces a formula $\varphi \in \Theta^{*}$ only if $[w]$ forces $\varphi$.

In the quotient model, $\left[w_{1}\right]$ will be less than $\left[w_{2}\right]$ only if $w_{1} \precsim w_{2}$. As $\precsim$ is a preorder, it follows easily that this ordering is well-defined. If $R$ is the reachability relation on the canonical model, then $\left[w_{1}\right]$ is reachable from $\left[w_{2}\right]$ in the quotient model only if there is some $w_{1}^{\prime} \in\left[w_{1}\right]$ and $w_{2}^{\prime} \in\left[w_{2}\right]$ such that $w_{1}^{\prime} R w_{2}^{\prime}$. The equivalence of $\simeq$ ensures that reachability relation is well-defined. If $I$ is the interpretation of atoms in the canonical model and $w=(Q, \Delta, q)$, then an atom $A$ will be placed in a world $[w]$ only if $A$ at $q \in \Delta$. Since a morphism between pcontexts always preserves points, the interpretation function is also well-defined.

Finally, the evaluation of a world $[w]$ in the canonical model will be partial. It is defined only if the point of $w$ is in $P$, and in that case the evaluation of $[w]$ is the point of $w$. Please note that morphisms between pcontexts always fixes elements in $P$, and therefore the evaluation is also welldefined. Moreover, partiality is essential for the well-definedness of the evaluation as a morphism of pcontexts may not preserve places other than those in $P$.

We start by defining the quotient model formally, and show that this is indeed a birelational model.

Definition 30 (Quotient Canonical Model) Given a finite set of places $P$ and two finite sets of pure formulae $\Gamma, \Theta \subseteq \operatorname{Frm}(P)$, let $\mathcal{W}_{\text {can }}=(W, \leq, R, I$, Eval $)$ be the $\Gamma$-prime and $(P, \Theta)$-bounded canonical birelational model with set of places $P l s$. The quotient model of $\mathcal{W}_{\text {can }}$ has set of places $P$, and is defined to be the quintuple $\left(W_{/ \simeq}, \leq^{\prime}, R^{\prime}, I^{\prime}, E v a l^{\prime}\right)$, where

1. The set $W / \simeq$ is the set of the equivalence classes generated by the relation $\simeq$ on $W$.

2. The binary relation $\leq^{\prime}$ is defined as: $\left[w_{1}\right] \leq^{\prime}\left[w_{2}\right]$ if and only if $w_{1} \precsim w_{2}$. 
3. The binary relation $R^{\prime}$ is defined as: $\left[w_{1}\right] R^{\prime}\left[w_{2}\right]$ if and only if there exists $w_{1}^{\prime} \in\left[w_{1}\right]$ and $w_{2}^{\prime} \in\left[w_{2}\right]$ such that $w_{1}^{\prime} R w_{2}^{\prime}$.

4. The function $I^{\prime}:$ Atoms $\rightarrow \operatorname{Pow}\left(W_{/ \bumpeq}\right)$ is defined as:

$$
I^{\prime}(A) \stackrel{\text { def }}{=}\{[w]: w \in I(A)\}
$$

5. The partial function $E v a l^{\prime}: W_{/ \simeq} \rightarrow P$ is defined as:

$$
\operatorname{Eval}^{\prime}([w]) \stackrel{\text { def }}{=} \begin{cases}p & \text { if } w=(Q, \Delta, p) \text { and } p \in P ; \\ \text { not defined } & \text { otherwise. }\end{cases}
$$

As we discussed before, $\leq^{\prime}, R^{\prime}, I^{\prime}$ and Eval ${ }^{\prime}$ in the quotient model are well-defined. The quotient model is a finite birelational model, as stated in the following proposition.

Proposition 31 (Birelational Preservation) Let $\mathcal{W}_{\text {can }}=(W, \leq, R, I$, Eval $)$ be the $\Gamma$-prime and $(P, \Theta)$-bounded canonical birelational model with set of places Pls. Let $\mathcal{W}_{/ \simeq}=\left(W_{/ \simeq}, \leq^{\prime}\right.$ , $\left.R^{\prime}, I^{\prime}, E v a l^{\prime}\right)$ be the quotient model of $\mathcal{W}_{c a n}$. Then $\mathcal{W}_{/ \bumpeq}$ is a finite birelational model with set of places $P$.

Proof The finiteness of $\mathcal{W} / \simeq$ follows from Lemma 29. The properties required by Definition 7 are easily checked, a particular attention is needed only to prove the transitivity for $R^{\prime}$. Since this case is tricky but not illuminating, we refer to the companion technical report [11]. As an example, we show uniqueness for Eval' here.

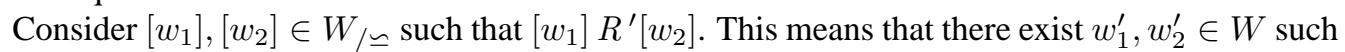
that $w_{1} \simeq w_{1}^{\prime} R w_{2}^{\prime} \simeq w_{2}$. Assume that $\left[w_{1}\right] \downarrow q$ and $\left[w_{2}\right] \downarrow q$. Then $w_{1}^{\prime} \downarrow q$ and $w_{2}^{\prime} \downarrow q$ in $\mathcal{W}_{\text {can }}$. The uniqueness property in $\mathcal{W}_{\text {can }}$ says that $w_{1}^{\prime}=w_{2}^{\prime}$. Hence $w_{1} \simeq w_{1}^{\prime} \simeq w_{2}$. We conclude $\left[w_{1}\right]=\left[w_{2}\right]$ as required by uniqueness.

We will show that a world $w$ forces a formula in $\Theta^{*}$ in the canonical birelational model if and only if $[w]$ forces the formula in the quotient model. For this, we will need the following proposition which states that given worlds $w_{1} \precsim w_{2}$ in the canonical model, if $w_{1}$ forces a formula in $\Theta^{*}$ then so does $w_{2}$ :

Proposition 32 (Forcing Preservation Under Morphisms) Given a finite set of places $P$ and two finite sets of pure formulae $\Gamma, \Theta \subseteq \operatorname{Frm}(P)$, let $\mathcal{W}_{\text {can }}=(W, \leq, R, I, E v a l)$ be the $\Gamma$-prime and $(P, \Theta)$-bounded canonical birelational model. Let $=_{\mathcal{W}}$ be the extension of interpretation $I$ to formulae. Then for every $w_{1}, w_{2} \in W$, and $\varphi \in \Theta^{*}$ :

1. If $w_{1} \precsim w_{2}$ then $w_{1} \models \mathcal{W} \varphi$ implies $w_{2} \models \mathcal{W} \varphi$.

2. If $w_{1} \simeq w_{2}$, then $w_{1} \models \mathcal{W} \varphi$ if and only if $w_{2} \models \mathcal{W} \varphi$.

Proof We prove the first point as the second one is straightforward consequence of the first one. Consider $w_{1}, w_{2} \in W$ such that $w_{1} \precsim w_{2}$. This means that $w_{1}=\left(Q_{1}, \Delta_{1}, q_{1}\right)$ and $w_{2}=$ $\left(Q_{2}, \Delta_{2}, q_{2}\right)$ where $\left(Q_{i}, \Delta_{i}\right)$ are $\Gamma$-prime and $(P, \Theta)$-bounded contexts for $i=1,2$. Moreover, there is a morphism $f: Q_{1} \rightarrow Q_{2}$ such that $f\left(q_{1}\right)=q_{2}$.

Assume that $w_{1} \models_{\mathcal{W}} \varphi$ for some $\varphi \in \Theta^{*}$. This means from the definition of canonical birelational model that $\varphi$ at $q_{1} \in \Delta_{1}$. Since $f$ is a morphism from $w_{1}$ to $w_{2}$, we get that $\varphi$ at $q_{2} \in \Delta_{2}$. Once again, we get from the definition of canonical birelational model that $w_{2} \models_{\mathcal{W}} \varphi$. 
We are now ready to prove that if the world $w$ in the canonical birelational model forces $\varphi \in \Theta^{*}$, then the world $[w]$ in the quotient model also forces $\varphi$, and vice-versa.

Lemma 33 (Quotient Forcing Preservation) Given a finite set of places $P$ and two finite sets of pure formulae $\Gamma, \Theta \subseteq \operatorname{Frm}(P)$, let $\mathcal{W}_{\text {can }}=(W, \leq, R, I, E v a l)$ be the $\Gamma$-prime and $(P, \Theta)-$ bounded canonical birelational model. Let $\mathcal{W}_{/ \simeq}=\left(W_{/ \simeq}, \leq^{\prime}, R^{\prime}, I^{\prime}, E_{v a l}\right)$ be the quotient model of $\mathcal{W}_{\text {can }}$. Let $=_{\mathcal{W}}$ and $\models / \simeq$ extend the interpretations $I$ and $I^{\prime}$ to formulae respectively. Then, for every $\varphi \in \Theta^{*}$ and $w \in W$ :

$$
w \models_{\mathcal{W}} \varphi \text { if and only if }[w] \models / \simeq \varphi .
$$

Proof The proof proceeds by induction on the structure of the formula $\varphi \in \Theta^{*}$.

Base case. The lemma is verified on $T$, and on $\perp$ by definition. Consider now the case when $\varphi=A \in$ Atoms. Then $w=\mathcal{W} A$ means $w=(Q, \Delta, q)$ for some $Q, \Delta, q$ and $A$ at $q \in \Delta$. Hence, $[w] \in I^{\prime}(A)$, and therefore $[w] \models / \bumpeq A$. The other direction is similar.

Induction hypothesis. We consider a formula $\varphi \in \Theta^{*}$, and we assume that the lemma holds for each sub-formula of $\varphi$ that is in $\Theta^{*}$. We will proceed by cases on the structure of $\varphi$. For the sake of clarity, we will just consider the case of modalities $\square$ and @ $p$. The other cases can be dealt with similarly. Please note that as $\Theta^{*}$ is closed under sub-formulae, the induction hypothesis can be applied to all sub-formulae of $\varphi$. Moreover, by definition, if $w_{1}=\left(Q_{1}, \Delta_{1}, q_{1}\right)$ and $w_{2}=$ $\left(Q_{2}, \Delta_{2}, q_{2}\right)$ are two worlds in $W$ such $w_{1} \leq w_{2}$ then $w_{1} \precsim w_{2}$.

Case $\varphi=\square \varphi_{1}$. Let $w \models_{\mathcal{W}} \varphi$. We need to show that $[w] \models / \simeq \square \varphi_{1}$. Consider $\left[w_{1}\right] \geq^{\prime}[w]$ and $\left[w_{2}\right] R^{\prime}\left[w_{1}\right]$. It suffices to show that $\left[w_{2}\right] \models / \simeq \varphi_{1}$. The hypothesis $\left[w_{2}\right] R^{\prime}\left[w_{1}\right] \geq^{\prime}[w]$ means that $w_{1} \succsim w$ and $w_{2} \simeq w_{3} R w_{4} \simeq w_{1}$ for some worlds $w_{3}, w_{4} \in W$. We get that $w_{4} \succsim w$ as $\precsim$ is a preorder .

We have $w_{4} \succsim w$, and hence $w_{4} \models \mathcal{W} \square \varphi_{1}$ by Proposition 32. By definition of forcing, $w_{3} \models_{\mathcal{W}} \varphi_{1}$. Therefore $w_{2} \models_{\mathcal{W}} \varphi_{1}$ by Proposition 32. The induction hypothesis says that $\left[w_{2}\right] \models / \simeq \varphi_{1}$, and so we conclude $[w] \models / \simeq \square \varphi_{1}$.

For the other direction, let $[w] \models / \simeq \square \varphi_{1}$. Consider $w_{1} \geq w$ and $w_{2} R w_{1}$. We have to show that $w_{2}=\varphi_{1}$.

We have $w_{1} \succsim w$, and hence $\left[w_{1}\right] \geq[w]$. We also have by the definition of the quotient model that $\left[w_{2}\right] R^{\prime}\left[w_{1}\right]$. Therefore, as $[w] \models / \simeq \square \varphi_{1}$, we get that $\left[w_{2}\right] \mid=/ \simeq \varphi_{1}$. Hence $w_{2} \models \mathcal{W} \varphi_{1}$ by induction hypothesis. We conclude that $w=\mathcal{W} \square \varphi_{1}$.

Case $\varphi=\varphi_{1} @ q$. As $\varphi \in \Theta^{*}$ and $\Theta^{*} \subseteq \operatorname{Frm}(P)$, we get that $q \in P$.

Now, if $w \models \mathcal{W} \varphi$ then there exists $w_{1} R w$ such that $w_{1} \models \mathcal{W} \quad \varphi_{1}$ and $w_{1} \downarrow q$. We have $\left[w_{1}\right] R^{\prime}[w]$ by definition of quotient model. As $q \in P$, we also have $\left[w_{1}\right] \downarrow q$. Therefore, $[w] \models / \simeq \varphi_{1} @ q$.

For the other direction, let $[w] \models / \simeq \varphi$. Then there exists $\left[w_{1}\right] R^{\prime}[w]$ such that $\left[w_{1}\right] \models / \simeq \varphi_{1}$, and $\left[w_{1}\right] \downarrow q$. This means that there are $w_{1}^{\prime}$ and $w^{\prime}$ such that $w_{1} \bumpeq w_{1}^{\prime} R w^{\prime} \simeq w$, and $w_{1} \models_{\mathcal{W}}$ $\varphi_{1}$ by induction hypothesis. Furthermore, $w_{1} \downarrow q$ and $w_{1}^{\prime} \downarrow q$. By Proposition 16, we get that $w_{1}^{\prime} \models \mathcal{W} \varphi_{1}$. Hence, by definition of forcing, $w^{\prime} \models \mathcal{W} \varphi_{1} @ q$. By Proposition 16 once again, $w \models \mathcal{W} \varphi_{1} @ q$.

As a result of Lemma 33, we have a way to going from a canonical model to an equivalent finite model. As shown above, the canonical model forces a formula if and only if its finite quotient does, and we get finite model property: 
Theorem 34 (Finite Model Property) Assume that $P$ is a finite set of places. If the judgement $\Gamma ; \Delta \vdash^{P} \varphi$ at $p$ is not provable, then there exists a finite birelational model $\mathcal{W}$ with set of places $P$, such that $\Gamma ; \Delta \vdash^{P} \varphi$ at $p$ is not valid in $\mathcal{W}$.

Proof We fix $\Theta \stackrel{\text { def }}{=}\{\square \psi ; \psi \in \Gamma\} \cup \Gamma \cup\{\psi: \psi$ at $q \in \Delta\} \cup \operatorname{PL}(\varphi) \cup\{p\}$. Consider the $\Gamma$-prime and $(P, \Theta)$-bounded canonical birelational model $\mathcal{W}_{c a n}$. From the proof of completeness in $\S 4$ there is a world of $\mathcal{W}_{\text {can }}$, say $w$, such that $w$ evaluates to $P$ and $w$ forces $\Gamma ; \Delta$ but not $\varphi$.

Consider the quotient $\mathcal{W}_{/ \bumpeq}$ of $\mathcal{W}_{\text {can }} . \mathcal{W}_{/ \simeq}$ is a finite birelational model and has set of places $P$. The world $[w]$ evaluates to $p$. Furthermore, as a consequence of Lemma 33, we can easily show that $[w]$ forces $\Gamma ; \Delta$ but not $\varphi$. Therefore, $\mathcal{W}_{/ \simeq}$ is the required finite counter-model.

Decidability is based on the usual Harrop criterion, cf. [14]: every finitely axiomatisable modal logic with the finite model property is decidable.

Corollary 35 (Decidability) The provability of the judgement $\Gamma ; \Delta \vdash^{P} \varphi$ at $p$ is decidable in the logic.

Proof Let $P^{\prime}$ be $\operatorname{PL}(\Gamma) \cup \operatorname{PL}(\Delta) \cup \operatorname{PL}(\varphi) \cup\{p\}$. By Proposition $26, \Gamma ; \Delta \vdash^{P} \varphi$ at $p$ if and only if $\Gamma ; \Delta \vdash P^{\prime} \varphi$ at $p$. As the function PL can be effectively computed, we just need to consider the judgement $\Gamma ; \Delta \vdash^{P^{\prime}} \varphi$ at $p$ for the decidability result.

We can enumerate all proofs in the logic in which the set of places considered is finite. Hence, we obtain an effective enumeration of all provable judgements. We can also effectively enumerate all finite birelational models, and effectively check whether the judgement $\Gamma ; \Delta \vdash^{P^{\prime}} \varphi$ at $p$ is refutable in a given finite birelational model. As a consequence of the finite model property proved above, $\Gamma ; \Delta \vdash^{P^{\prime}} \varphi$ at $p$ is refutable only if it is refutable in some finite birelational model. By performing these enumerations and checks simultaneously, we obtain an effective test for provability of $\Gamma ; \Delta \vdash P^{\prime} \varphi$ at $p$.

The procedure detailed in the corollary above would not have worked if we had used Kripke models instead of birelational models. This is because the finite model property fails for Kripke models. For example, consider the judgement ; $\square \neg \neg A$ at $p \vdash\{p\} \neg \neg \square$ A at $p$. We claim that this judgement is valid for every finite Kripke model.

Indeed, let $k$ be a Kripke state in some finite Kripke model $\mathcal{K}$ such that $(k, p) \models \square \neg \neg A$. Pick any $l \geq k$ in $\mathcal{K}$ such that $l$ is maximal with respect to the ordering of Kripke states. As $(k, p) \models \square \neg \neg A$, we get by definition that $(l, r) \models \neg \neg A$ for every place $r$ in the state $l$. From the semantics of implication and the fact that $l$ is a maximal state, it must be the case that $(l, r) \models A$ for every place $r$ in the state $l$. Again, as $l$ is maximal, we get $(l, p) \models \square A$. As the model is finite, there is always a maximal $l^{\prime}$ above any $k^{\prime} \geq k$, We conclude $(k, p) \models \neg \neg \square A$ from semantics of implication.

On the other hand, we showed that the judgement is not valid in the finite model $\mathcal{W}_{\text {exam }}$ in Example 9. The model $\mathcal{W}_{\text {exam }}$ has two worlds $w_{1}$ and $w_{2}$ such that $w_{1} \leq w_{2}, w_{1} R w_{2}, I(A)=$ $\left\{w_{2}\right\}, w_{1} \uparrow$ and $w_{2} \downarrow p$. As we discussed there, $w_{2} \models \square \neg \neg A$ and $w_{2} \not \models \neg \neg \square A$. As we mentioned before, this example is adapted from $[25,36]$.

\section{Related Work}

The logic we studied is an extension of the logic introduced in [17, 18]. In [17, 18], it was used as the foundation of a type system for a distributed $\lambda$-calculus by exploiting the proofs-as-terms and propositions-as-types paradigm. The proof terms corresponding to modalities have computational 
interpretation in terms of remote procedure calls (@p), commands to broadcast computations $(\square)$, and commands to use portable code $(\diamond)$. The authors also introduce a sequent calculus for the logic without disjunctive connectives and prove that it enjoys cut elimination. Although the authors demonstrate the usefulness of logic in reasoning about the distribution of resources, they do not have a corresponding model.

The proofs-as-terms and propositions-as-types paradigm has also been used in [38, 39, 22]. In [38], the logic studied is an intuitionistic modal logic derived from IS5, and the modalities have a spatial flavour. Specifically, Kripke states are taken to be nodes on a network. The connective $\square$ reflects the mobility of portable code, and $\diamond$ reflects the address of a fixed resources. The work in [39] extends $[38,17,18]$ to a lambda calculus for classical hybrid $S 5$ with network-wide continuations which arise naturally from the underlying classical logic. These continuations give a computational interpretation of theorems of classical hybrid S5. In [22], the relationship between modal logics and type systems for Grid computing is investigated. The objects with type $\square$ are interpreted as jobs that may be injected into the Gird and run anywhere. The main difference from $[39,38,17,18]$ is that the underlying logic is based on $S 4$ rather than $S 5$. Whereas $[39,38,17,18]$ assume all nodes are connected to all other nodes, networks may have a more refined accessibility relation.

From a logical point of view, the logic in this paper can be viewed as a hybrid modal logic $[1,2,4,5,6,28,29]$. A hybrid logic internalises the model in the logic by using modalities built from pure names. The original idea of internalising the model into formulae was proposed in [28, 29], and has been further investigated in $[1,2,4,5,6]$. This work has been mostly carried out in the classical setting. More recently, classical hybrid logic is combined with linear temporal logic in [26] and that logic accounts for both temporal and spatial aspects. Intuitionistic versions of hybrid logics were investigated in $[7,17,18]$.

There are several intuitionistic modal logics in the literature, and [36] is a good source on them. The modalities in [36] have a temporal flavour. The spatial interpretation was not recognised then. In [36], for example, the accessibility relation expresses the next step of a computation. The work in [7] extends the modal systems in [36] and creates hybrid versions of the modal systems by introducing nominals. These are a new kind of propositional symbols which project semantics into the logic. A natural deduction system for these hybrid systems along with a normalisation result is also given in [7]. A Kripke semantics along with a proof of soundness and completeness is also introduced.

The extension we gave to the logic in $[17,18]$ is a hybrid version of the intuitionistic modal system IS5 $[24,30,36]$. The modality @ $p$ internalises the model in the logic. In the modal system IS5, first introduced in [30], the accessibility relation among places is total. The main difference in the logic presented in $[7]$ and the logic in $[17,18]$ is that names in $[17,18]$ only occur in the modality $@ p$.

From the point of view of semantics, Kripke semantics were first introduced in [20] for intuitionistic first-order logic. Kripke semantics for intuitionistic modal systems were developed in $[12,24,27,35,36]$. Birelational models for intuitionistic modal logic were introduced independently in $[12,35,27]$. They are in general useful to prove the finite model property as demonstrated in $[25,36]$. The finite model property fails for Kripke semantics [36, 25], and an example for this was adapted in this paper.

Some other examples of work on logics for resources are separation logics [34] and BI, the logic of bunched implications [23, 32,33]. Separation logic is an extension of Hoare logic that permits reasoning about low-level imperative programs with shared mutable data structure. Formulae are extended by introducing a 'separating conjunction' whose subformulae are meant to hold for disjoint parts of the system, thus enabling a concise and flexible description of structures with controlled sharing. BI is the theoretical base to separation logics. While separation logic is based on particular 
storage models, BI describes resources more generally and its model theory is inspired by a primitive of resource composition.

BI is a substructural system which combines freely the propositional intuitionistic logic and the multiplicative fragment of propositional linear logic. Assertions are not in a sequence but in bunches: contexts with two combining operations, one reflected in the logic by the intuitionistic conjunction and the other by the multiplicative (linear) one. In [23, 32, 33], the authors give a Kripke model based on monoids. The formulae of the logic are the resources, and are interpreted as elements of the monoid. The monoidal operation is reflected in the logic by the multiplicative connective. The focus of this work is the sharing of resources and not their distribution.

BI-Loc, presented in [3], extends the logic of bunched implication by introducing a modality for locations. Its models are resource trees: node-labelled trees in which nodes contain resources belonging to a monoid. Every label gives rise to a corresponding logical modality which precisely indicates the location where a formula holds. Although BI-Loc offers a separation operator to express properties holding in different parts of the system, its propositional fragment cannot state properties verified in an unspecified node or in every node of the system. To fill this gap, authors introduce quantifications on locations and paths. Validity is undecidable for the full BI-Loc with quantifications, but it becomes decidable by avoiding the multiplicative (linear) implication.

The Logic of Bunched Implications has been recently extended in [31] with modalities, in a Hennessy-Milner style [15]. The new logic, MBI, is suitable to express properties of concurrent systems specified in a calculus of resources and processes. This gives a modal logic and a semantics that combines Kripke relational semantics with BI Kripke monoid semantics. A similar approach is presented in [8] where a Spatial Logic models the asynchronous $\pi$-calculus [21]. The logic is developed in classical settings and lacks a notion of resources. The main aim of spatial logic is to describe the behaviour and the spatial structure of concurrent systems. The logic is modal in space and in time, and a formula describes a property of a particular part of a concurrent system at a particular time.

Locations can be added to Spatial Logic along the lines of [9] which gives a modal logic based on Ambient Calculus [10]. Ambients are intended as locations, and there is a modality $m$ [_] for every ambient name $m$ which specifies the location where a property holds. These spatial modalities have an intensional flavour and 'hybridise' spatial logics as the modality @ $p$ 'hybridises' IS5 in the current paper. However, the locations in Ambient logic unlike this paper have an intensional hierarchy which is reflected in the logic by having nested formulae like $m[n[T]]$.

\section{Conclusions and Future Work}

We studied the hybrid modal logic presented in [17, 18], and extended the logic with disjunctive connectives. Formulae in the logic contain names, also called places. The logic is useful to reason about placement of resources in a distributed system. We gave two sound and complete semantics for the logic.

In one semantics, we interpreted the judgements of the logic over Kripke-style models [20]. Typically, Kripke models [20] consist of partially ordered Kripke states. In our case, each Kripke state has a set of places, and different places satisfy different formulae. Larger Kripke states have larger sets of places, and the satisfaction of atoms corresponds to the placement of resources. The modalities of the logic allow formulae to be satisfied in a named place (@p), some place $(\diamond)$ and every place $(\square)$. The Kripke semantics can be seen as an instance of hybrid IS5 [24, 30, 7, 36].

In the second semantics, we interpreted the judgements over birelational models $[12,35,27,36]$. 
Typically, birelational models have a set of partially ordered worlds. In addition to the partial order, there is also a reachability relation amongst worlds. In order to interpret the modality @ $p$ in the system, we also introduced a partial evaluation function on the set of worlds. The hybrid nature of the logic presented difficulties in the proof of soundness. The difficulties are addressed using a mathematical construction that creates a new model from a given one. The set of worlds in the constructed model is the union of two sets. One of these sets is the reachability relation, and the worlds in the second set witness the existential and universal properties.

As in the case of intuitionistic modal systems [12, 35, 24, 27, 36], we demonstrated that the birelational models introduced here enjoy the finite model property: a judgement is not provable in the logic if and only if it is refutable in some finite model. The finite model property allowed us to conclude decidability. The partiality of the evaluation function was essential in the proof of the finite model property.

As future work, we are considering other extensions of the logic. A major limitation of the logic presented in $[17,18]$ is that if a formula $\varphi$ is validated at some named place, say $p$, then the formula $\varphi @ p$ can be inferred at every other place. Similarly, if $\nabla \varphi$ or $\square \varphi$ can be inferred at one place, then they can be inferred at any other place. In a large distributed system, we may want to restrict the rights of accessing information in a place. This can be done by adding an accessibility relation as is done in the case of other intuitionistic modal systems $[36,7]$. We are currently investigating if the proof of the finite model property can be adapted to the hybrid versions of other intuitionistic modal systems. We are also investigating the computational interpretation of these extensions. This would result in extensions of $\lambda$-calculus presented in $[17,18]$. We also plan to investigate adding temporal modalities to the logic. This will help us to reason about both space and time.

From a purely logical point of view, the meta-logic used in the paper to reason about soundness and completeness is classical. In order to obtain a full intuitionistic account for the logic, another line of investigation would be to consider categorical and/or topological semantics for the logic. This would allow us to obtain soundness and completeness results when the meta-logic is intuitionistic.

Acknowledgements. We thank Annalisa Bossi, Giovanni Conforti, Valeria de Paiva, Matthew Hennessy, and Bernhard Reus for interesting and useful discussions. We also thank the anonymous referees for insightful and valuable comments.

\section{References}

[1] C. Areces and P. Blackburn. Bringing them all together. Journal of Logic and Computation, 11(5):657-669, 2001.

[2] C. Areces, P. Blackburn, and M. Marx. Hybrid logics: Characterization, interpolation and complexity. Journal of Symbolic Logic, 66:997-1010, 2001.

[3] N. Biri and D. Galmiche. A separation logic for resource distribution. In FSTTCS'03, volume 2914 of LNCS, pages 23-37. Springer Verlag, 2003.

[4] P. Blackburn. Internalizing labelled deduction. Journal of Logic and Computation, 10:137$168,2000$.

[5] P. Blackburn. Representation, reasoning, and relational structures: a hybrid logic manifesto. Logic Journal of the IGPL, 8:339-365, 2000. 
[6] P. Blackburn and J. Seligman. What are hybrid languages? In M. Kracht, M. de Rijke, H. Wansing, and M. Zakharyaschev, editors, Advances in modal logic, volume 1, pages 41-62. CSLI, 1996.

[7] T. Braüner and V. de Paiva. Towards constructive hybrid logic (extended abstract). In Elec. Proc. of Methods for Modalities 3, 2003.

[8] L. Caires and L. Cardelli. A spatial logic for concurrency (part I). In TACS'01, volume 2215 of LNCS, pages 1-37. Springer Verlag, 2001.

[9] L. Cardelli and A.D. Gordon. Anytime, anywhere. Modal logics for mobile ambients. In POPL'00, pages 365-377. ACM Press, 2000.

[10] L. Cardelli and A.D. Gordon. Mobile ambients. Theoretical Computer Science, Special Issue on Coordination, 240(1):177-213, 2000.

[11] R. Chadha, D. Macedonio, and V. Sassone. A hybrid intuitionistic logic: Semantics and decidability. (extended version). Computer Science Report 2005:07, University of Sussex, 2005.

[12] W. B. Ewald. Time, Modality and Intuitionism. PhD thesis, University of Oxford, 1978.

[13] J.-Y. Girard. Proofs and Types. Cambridge University Press, 1989.

[14] R. Harrop. On the existence of finite models and decision procedures for propositional calculi. In Proc. of Cambridge Philosophical Society, volume 54, pages 1-13, 1958.

[15] M. Hennessy and R. Milner. Algebraic laws for nondeterminism and concurrency. Journal of the ACM, 32(1):137-161, 1985.

[16] M. Hennessy and J. Riely. Resource access control in systems of mobile agents. Information and Computation, 173:82-120, 2002.

[17] L. Jia and D. Walker. Modal proofs as distributed programs. Technical Report TR-671-03, Princeton University, 2003.

[18] L. Jia and D. Walker. Modal proofs as distributed programs (extended abstract). In ESOP'04, volume 2986 of LNCS, pages 219-233. Springer Verlag, 2004.

[19] S.A. Kripke. Semantical analysis of modal logic I: Normal modal propositional calculi. In Zeitschrift für Mathematische Logik und Grundlagen der Mathematik, volume 9, pages 67-96, 1963.

[20] S.A. Kripke. Semantical analysis of intuitionistic logic, I. In Proc. of Logic Colloquium, Oxford, 1963, pages 92-130. North-Holland Publishing Company, 1965.

[21] R. Milner, J. Parrow, and D. Walker. A calculus of mobile processes, parts I and II. Information and Computation, 100(1):1-77, 1992.

[22] J. Moody. Modal logic as a basis for distributed computation. Technical Report CMU-CS-03194, Carnegie Mellon University, 2003.

[23] P.W. O'Hearn and D. Pym. The logic of bunched implications. Bulletin of Symbolic Logic, 5(2):215-244, 1999. 
[24] H. Ono. On Some Intuitionistic Modal Logics, volume 13, pages 687-722. Publications of RIMS, Kyoto University, 1977.

[25] H. Ono and N.-Y. Suzuki. Relations between intuitionistic modal logics and intermediate predicate logics. Reports on Mathematical Logic, 22:65-87, 1988.

[26] D. Pattinson and B. Reus. A complete temporal and spatial logic for distributed systems. In Frontiers of Combining Systems (FroCoS), volume 3717 of LNAI, pages 122-137. SpringerVerlag, 2005.

[27] G. D Plotkin and C. P Stirling. Theoretical Aspects of Reasoning About Knowledge, chapter A Framework for Intuititionistic Modal Logic. J. Y. Halpern, 1986.

[28] A. Prior. Past, Present and Future. Oxford University Press, 1967.

[29] A. Prior. Papers on Time and Tense. Oxford University Press, 1968.

[30] A. N. Prior. Time and Modality. Oxford University Press, 1957.

[31] D. Pym and C. Tofts. A calculus and logic of resources and processes. Technical Report HPL-2004-170R1, HP Laboratories Bristol, 2005.

[32] D.J. Pym. The Semantics and Proof Theory of the Logic of the Logic of Bunched Implications, volume 26 of Applied Logic Series. Kluwer Academic Publishers, 2002.

[33] D.J. Pym, P.W. O'Hearn, and H. Yang. Possible worlds and resources: The semantics of BI. Theoretical Computer Science, 315(1):257-305, 2004.

[34] J. Reynolds. Separation logic: a logic for shared mutable data structures. In LICS'02, pages 55-74. IEEE Computer Society Press, 2002.

[35] G. Fisher Servi. Semantics for a class of intuitionistic modal calculi. In M. L. dalla Chiara, editor, Italian Studies in the Philosophy of Science, pages 59-72. Reidel Publishing Company, 1981.

[36] A.K. Simpson. The Proof Theory and Semantics of Intuitionistic Modal Logic. PhD thesis, University of Edinburgh, 1994.

[37] D. van Dalen. Logic and Structure. Springer Verlag, 4th extended edition, 2004.

[38] T. Murphy VII, K. Crary, R. Harper, and F. Pfenning. A symmetric modal lambda calculus for distributed computing. In Proc. of LICS'04, pages 286-295. IEEE Press, 2004.

[39] T. Murphy VII, R. Harper, and K. Crary. Distributed control flow with classical modal logic. In CSL'05, volume 3634 of LNCS, pages 51-69. Springer Verlag, 2005. 